\title{
The Synchronous Activity of Lateral Habenular Neurons Is Essential for Regulating Hippocampal Theta Oscillation
}

\author{
Hidenori Aizawa, ${ }^{1,2}$ Shin Yanagihara, ${ }^{1}$ Megumi Kobayashi, ${ }^{1}$ Kazue Niisato, ${ }^{1}$ Takashi Takekawa, ${ }^{1}$ Rie Harukuni, ${ }^{1}$ \\ Thomas J. McHugh, ${ }^{1,3}$ Tomoki Fukai, ${ }^{1,3}$ Yoshikazu Isomura, ${ }^{1,4}$ and Hitoshi Okamoto ${ }^{1,3}$ \\ ${ }^{1}$ RIKEN Brain Science Institute, Wako, Saitama 351-0198, Japan, ${ }^{2}$ Tokyo Medical and Dental University Medical Research Institute, Bunkyo-ku, Tokyo \\ 113-8510, Japan, ${ }^{3}$ Core Research for Evolutional Science and Technology, Japan Science and Technology Agency, Chiyoda-ku, Tokyo 102-0075, Japan, and \\ ${ }^{4}$ Tamagawa University Brain Science Institute, Machida, Tokyo, 194-8610, Japan
}

Lateral habenula $(\mathrm{LHb})$ has attracted growing interest as a regulator of serotonergic and dopaminergic neurons in the CNS. However, it remains unclear how the LHb modulates brain states in animals. To identify the neural substrates that are under the influence of LHb regulation, we examined the effects of rat LHb lesions on the hippocampal oscillatory activity associated with the transition of brain states. Our results showed that the LHb lesion shortened the theta activity duration both in anesthetized and sleeping rats. Furthermore, this inhibitory effect of LHb lesion on theta maintenance depended upon an intact serotonergic median raphe, suggesting that LHb activity plays an essential role in maintaining hippocampal theta oscillation via the serotonergic raphe. Multiunit recording of sleeping rats further revealed that firing of LHb neurons showed significant phase-locking activity at each theta oscillation cycle in the hippocampus. LHb neurons showing activity that was coordinated with that of the hippocampal theta were localized in the medial LHb division, which receives afferents from the diagonal band of Broca (DBB), a pacemaker region for the hippocampal theta oscillation. Thus, our findings indicate that the DBB may pace not only the hippocampus, but also the LHb, during rapid eye movement sleep. Since serotonin is known to negatively regulate theta oscillation in the hippocampus, phase-locking activity of the LHb neurons may act, under the influence of the DBB, to maintain the hippocampal theta oscillation by modulating the activity of serotonergic neurons.

\section{Introduction}

Learning needs coordinated activation of the specific brain regions that process sensory information. Theta rhythm is the oscillatory activity that underlies communication between the neural structures. Specifically, theta oscillation in the hippocampus (HPC) appears both in the awake and rapid eye movement (REM) sleep state, and has been implicated in modifying synaptic plasticity for memory formation (Buzsáki, 2002). Indeed, synchronous firing of the hippocampal neurons and theta oscillation predicts strength in memory formation (Rutishauser et al., 2010). Theta oscillation also coordinates the neural activity of distant brain regions such as amygdala, hippocampus, and prefrontal cortex during

Received Sept. 12, 2012; revised April 10, 2013; accepted April 15, 2013.

Author contributions: H.A. and H.O. designed research; H.A., S.Y., M.K., K.N., R.H., T.J.M., and Y.I. performed research; H.A., T.T., and T.F. analyzed data; H.A. and H.O. wrote the paper.

The authors declare no conflict of interests.

This research was supported by grants from RIKEN Brain Science Institute, Core Research for Evolutional Science and Technology of Japan Science and Technology Agency, a Grant-in-Aid for Scientific Research on Priority Areas (KAKENHI20021034), Scientific Research on Innovative Areas (KAKENHI21115521), and Scientific Research (B) (KAKENHI19300115) from the Ministry of Education, Culture, Sports, Science and Technology (MEXT) to H.0.; and by funds from Narishige Neuroscience Research Foundation, Mitsui Life Social Welfare Foundation, Takeda Science Foundation, Naito Foundation, Japan Health Foundation, and Uehara Memorial Foundation, and a Grant-in-Aid for Young Scientists (B) (KAKENHI24700350) from MEXT to H.A. We thank the members of our laboratories and Dr. Joshua P. Johansen for valuable discussions related to this work, as well as Kumi Ishii and Yuka Takeuchi for technical support.

Correspondence should be addressed to Hitoshi Okamoto, RIKEN Brain Science Institute, Wako, Saitama 3510198, Japan. E-mail: hitoshi@brain.riken.jp.

DOI:10.1523/JNEUROSCI.4369-12.2013

Copyright $\odot 2013$ the authors $\quad 0270-6474 / 13 / 338909-13 \$ 15.00 / 0$ behaviors contingent with emotional experiences such as anxiety (Adhikari et al., 2010) and fear (Seidenbecher et al., 2003). In support of a role for the modification of theta oscillation in learning, it was recently shown that increased synchrony of these theta activities is associated with impaired extinction recall of fear memory under the influence of diffuse serotonergic modulation (Narayanan et al., 2011). These facts indicate that theta oscillation plays a central role in memory formation, and changing the theta duration could modulate the efficiency of memory formation.

Habenula is a diencephalic nucleus conserved across vertebrates (Aizawa et al., 2011). It is subdivided into the medial and lateral nucleus according to the distinct composition of neurotransmitters and neural connectivity. Among these two components, the lateral habenula ( $\mathrm{LHb}$ ) has drawn considerable attention recently based on findings that this region could contribute to the regulation of dopaminergic and serotonergic activities (Wang and Aghajanian, 1977; Nishikawa and Scatton, 1985; Christoph et al., 1986; Nishikawa et al., 1986; Matsumoto and Hikosaka, 2007) and play a critical role in cognitive functions such as attention (Lecourtier and Kelly, 2005). In fact, transient stimulation of the LHb has an inhibitory effect on both dopaminergic (Christoph et al., 1986) and serotonergic neurons (Wang and Aghajanian, 1977), and its altered activity was implicated recently in the pathophysiology of psychiatric disorders such as depression (Sartorius et al., 2010; Li et al., 2011) and schizophrenia (Ellison, 1994; Lecourtier et al., 2004). Despite the functional significance, it remains unclear how the LHb might modulate such animal behaviors. 
$\mathrm{LHb}$ projects to the serotonergic raphe nuclei, which have been implicated in the negative modulation of hippocampal theta oscillation (Vertes et al., 1994). Considering that lesions of fiber bundles including the rat $\mathrm{LHb}$ efferents led to a reduced hippocampal theta (Valjakka et al., 1998), it is reasonable to hypothesize that theta oscillation in the hippocampus could mediate modulation of animal behaviors via neural activity in the LHb.

Here we report, for the first time, that intact LHb activity is needed to maintain hippocampal theta oscillation, and that more than half of the LHb neurons show phase-locking activity toward the oscillation. This specific neuronal activity is localized in the medial $\mathrm{LHb}$ division, which receives afferents preferentially from the diagonal band of Broca (DBB), a pace-making region of the hippocampal theta oscillation (Vertes and Kocsis, 1997). These findings suggest that LHb may mediate the regulation of theta oscillation in the hippocampus under the influence of the DBB.

\section{Materials and Methods}

\section{Animal preparation}

All experiments were performed in accordance with the Animal Experiment Plan approved by the Animal Experiment Committee of RIKEN. Adult Long-Evans rats (250-300 g, male; Japan SLC) were maintained throughout the experiments on a $12 \mathrm{~h}$ light/dark cycle schedule with lights on during the day and handled twice briefly before the primary surgery. For the head-restrained preparation, primary surgery for placing a sliding head-attachment and reference electrodes was conducted as described previously (Isomura et al., 2009). At the same time, we placed the Teflon-coated silver electrodes above the parietal (1.0 anterior, 2.5 $\mathrm{mm}$ lateral of bregma) and occipital (1.0 posterior, $3.5 \mathrm{~mm}$ lateral of bregma) cortices for cortical electroencephalography (EEG), and in the trapezius muscle for electromyography (EMG) (Ambrosini et al., 1993). After recovery from this primary surgery, animals were habituated to a head-restraint apparatus for $6 \mathrm{~d}$ over 2 weeks $(6-8 \mathrm{~h}$ in a day) so that they could sleep in that situation. The day before the electrophysiological recordings, the rats were subjected to the second surgery under isoflurane anesthesia, whereby two tiny holes were made in the skull and dura mater (3.6 $\mathrm{mm}$ posterior, $1.5 \mathrm{~mm}$ lateral of bregma for the $\mathrm{LHb}$; $4.5 \mathrm{~mm}$ posterior, $4.5 \mathrm{~mm}$ lateral of bregma for the hippocampus). The hole was covered with silicon sealant until the recording experiment commenced.

For recording neural activity in the sleeping rats with the LHb lesion, the animals received a sham or lesion procedure as described below and were then implanted with a custom-made microdrive array consisting of four independently adjustable tetrodes, as described previously (McHugh et al., 1996), targeted to CA1 (stereotaxic coordinates from bregma: $3.0 \mathrm{~mm}$ lateral; $3.6 \mathrm{~mm}$ posterior; $2.0 \mathrm{~mm}$ deep from the pia mater).

\section{Electrophysiological recordings}

For the head-restrained procedure, we obtained extracellular multiunit recordings from neurons through stainless wire tetrode (California Fine Wire) or custom-made 16-channel silicon probes (Neuronexus) tilted laterally by $10^{\circ}$ and placed $3.8-4.5 \mathrm{~mm}$ deep for LHb and $2.0-2.2 \mathrm{~mm}$ deep for the hippocampal CA1 area (sample, $20 \mathrm{kHz}$; final gain, 2000; original bandpass filter, $0.5 \mathrm{~Hz}$ to $10 \mathrm{kHz}$ for the hippocampus, and 300 $\mathrm{Hz}$ to $10 \mathrm{kHz}$ for the $\mathrm{LHb}$ ) connected to a hard-disk recorder (DataMax II, R.C. Electronics). We recorded unit activities from $\mathrm{LHb}$ at a narrower frequency band to maximize the dynamic range for unit activity and at the expense of neural information such as local field potential (LFP), because the unit amplitude in LHb was smaller than that in the hippocampus. The electrodes for the hippocampus were advanced in $10 \mu \mathrm{m}$ increments to target the CA1 pyramidal cell layer using a fine micromanipulator (Model 1760-61, David Kopf Instruments) on a stereotactic frame

(SR-8N, Narishige) until the magnitude of the ripples did not increase further. We simultaneously recorded the cortical activity by surface EEG electrodes or tetrodes in the visual cortex $(4.5 \mathrm{~mm}$ posterior, $4.5 \mathrm{~mm}$ lateral of bregma, and $0.8-1.2 \mathrm{~mm}$ in depth). Behavioral states were also monitored by video capturing the frontal view of the animals (60 frames/s) synchronously with recordings of the hippocampal and cortical activity and EMG ( $2 \mathrm{kHz}$; AQ-VU, TEAC).

For the freely moving preparation, we attached the headstage preamplifier (HS-36-LED, Neuralynx) to the microdrive connector, and then placed the rat in the home cage. Electrodes were slowly advanced over $2 \mathrm{~d}$ to reach the CA1 pyramidal cell layer by the same procedure as described for the head-restrained procedure.

For juxtacellular recordings, the animals were anesthetized with urethane $(1.25 \mathrm{~g} / \mathrm{kg})$ and supplemental doses of ketamine and xylazine $(20$ and $2 \mathrm{mg} / \mathrm{kg}$, respectively) as needed, and body temperature was retained with a heating pad, using the same coordinates as those used in the unanesthetized rats. Glass electrodes (BF150-75-10, Sutter Instrument) were prepared by a laser puller (P-2000, Sutter Instrument), and a blunt tip was created. The electrode was filled with $2 \%$ neurobiotin (Vector Laboratories) in $0.5 \mathrm{M} \mathrm{NaCl}(12-30 \mathrm{M} \Omega)$, and then inserted into the $\mathrm{LHb}$ with a microdrive (LSS-8000 Inchworm, Burleigh Instruments) installed on a $10^{\circ}$ tilted micromanipulator (Model 1760-61, David Kopf Instruments). Juxtacellular signals were amplified with an intracellular amplifier (IR-283, Cygnus Tech) and sampled at $20 \mathrm{kHz}$ (final gain, 1000; bandpass filter, $300-10 \mathrm{kHz}$ ) with a hard-disk recorder (DataMax II or LX-120, TEAC). Subsequently, neurobiotin was electroporated into recorded neurons with a positive current pulse (2-10 nA; $0.25 \mathrm{~s}$ duration, every $0.5 \mathrm{~s} ; 5-15 \mathrm{~min})$. Juxtacellular recordings were combined with extracellular recordings from the hippocampal CA1 pyramidal cell layer through a tetrode placed at $2.0-2.2 \mu \mathrm{m}$ deep (sampling, $20 \mathrm{kHz}$; final gain, 2000; original bandpass filter, $0.5 \mathrm{~Hz}$ to $10 \mathrm{kHz}$ ).

\section{Lesion surgery}

For electrolytic lesion, rats were anesthetized with $2 \%$ isoflurane and placed in the stereotactic apparatus (SR-8N, Narishige). A tiny hole was made in the skull and dura mater $(3.6 \mathrm{~mm}$ posterior, $1.5 \mathrm{~mm}$ lateral of bregma), and the stainless steel bipolar electrode (CBAPD75, FHC) tilted laterally by $10^{\circ}$ was placed $4.5 \mathrm{~mm}$ deep to target the LHb. We applied $1 \mathrm{~mA}$ of positive current for $30 \mathrm{~s}$ to make a lesion specifically in the LHb; sham-lesion cases received no current. For recording the LFP in the hippocampal CA1, the animals were implanted with a microdrive array consisting of four tetrodes at the same time. After a recovery period of $7 \mathrm{~d}$, the animals were subjected to either the same procedure as in the juxtacellular recording under urethane anesthesia or the freely moving preparation as described above.

To introduce a neurotoxic lesion of the serotonergic neurons, we injected 5,7-dihydroxytryptamine (5,7-DHT; creatinine sulfate, SigmaAldrich) into the median raphe as described previously (Limebeer et al., 2004). Briefly, 30 min before surgery, rats were pretreated with desipramine $(10 \mathrm{mg} / \mathrm{kg}$ mixed in saline and administered at a volume of $1 \mathrm{ml} / \mathrm{kg}$, i.p.; Sigma-Aldrich) to inhibit uptake of 5,7-DHT by noradrenergic neurons and prevent depletion of norepinephrine. After the rats were placed in the stereotaxic frame (Model 900, David Kopf Instruments), $4 \mu \mathrm{g}$ of 5,7 -DHT dissolved in a volume of $2 \mu \mathrm{l}$ of $0.1 \%$ ascorbic acid (SigmaAldrich) was injected into the median raphe $(7.5 \mathrm{~mm}$ posterior and 0.0 $\mathrm{mm}$ lateral from the bregma, $7.6 \mathrm{~mm}$ in depth) over a period of $4 \mathrm{~min}$. The animals were allowed to recover for 7-11 d before electrophysiological recording under urethane anesthesia as described above.

\section{Histology}

Under deep anesthesia, animals were perfused transcardially with cold saline followed by $4 \%$ paraformaldehyde in $0.1 \mathrm{~m}$ phosphate buffer $(\mathrm{PB})$, $\mathrm{pH}$ 7.4. Postfixed brains were sliced coronally into $50-\mu \mathrm{m}$-thick serial sections using a vibrating microtome (DTK-3000W, Dosaka EM). Neurobiotin-loaded neurons were visualized using the avidin-biotinperoxidase complex (Vectastain Elite ABC, Vector Laboratories) with diaminobenzidine and nickel (DAB-Ni), and sections were counterstained with neutral red or thionin. Neurons identified by juxtacellular labeling were mapped onto the schematic drawing of a coronal section showing the relative position of the cell body of the labeled neurons in the subnuclei of LHb by adjusting the relative size of each subnucleus. Subnuclei of LHb were identified according to the cell body size and cell density as described previously (Andres et al., 1999; Geisler et al., 2003; Aizawa et al., 2012). 

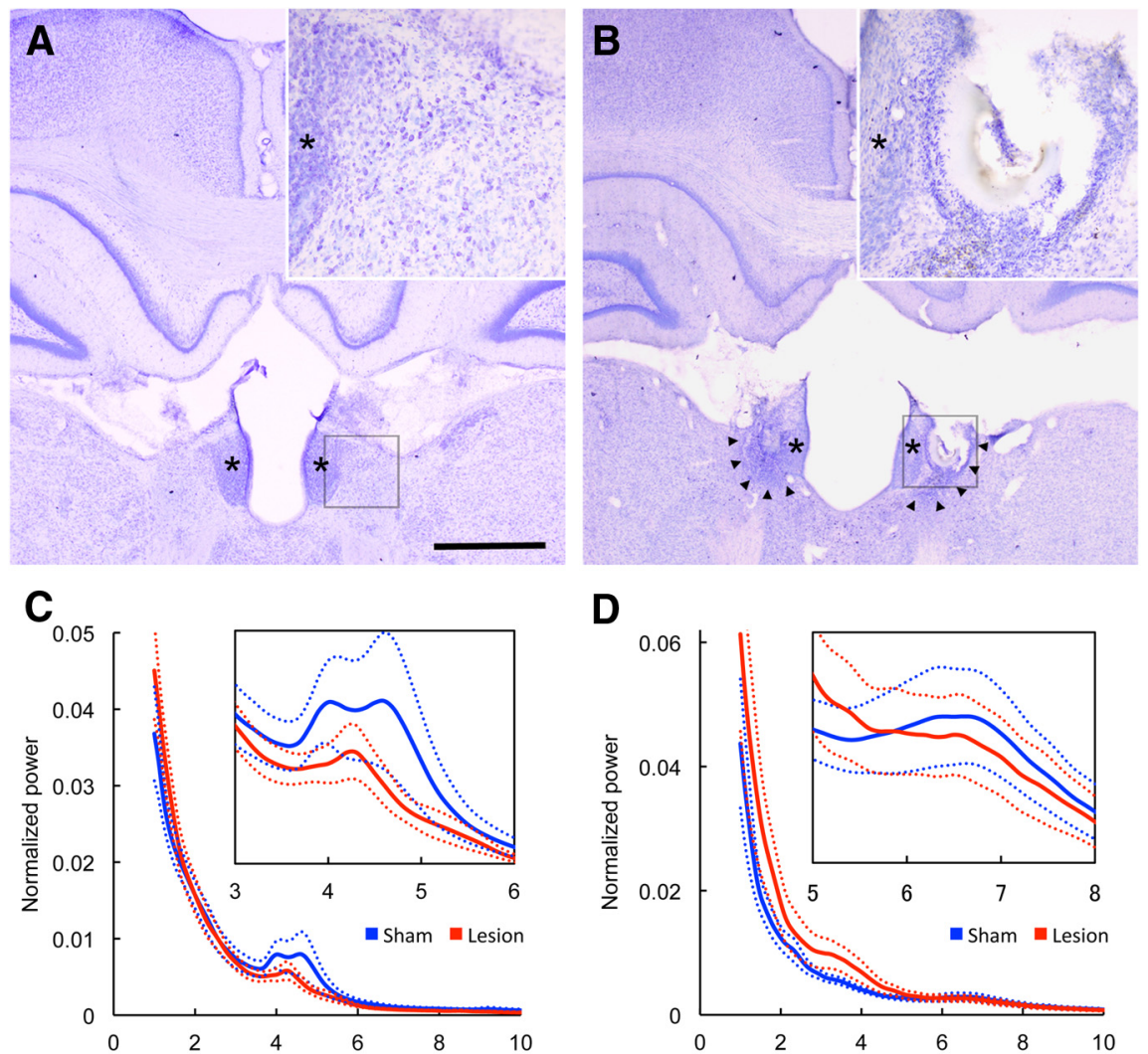

E

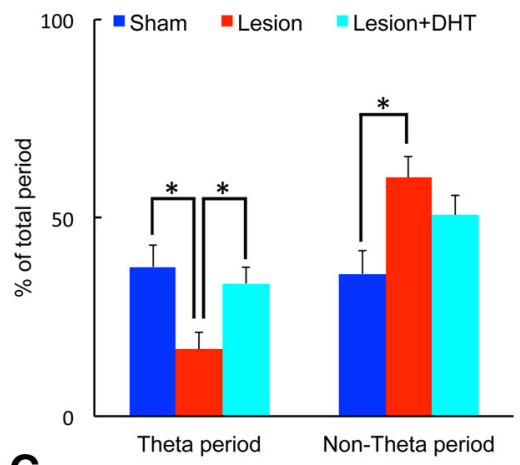

G

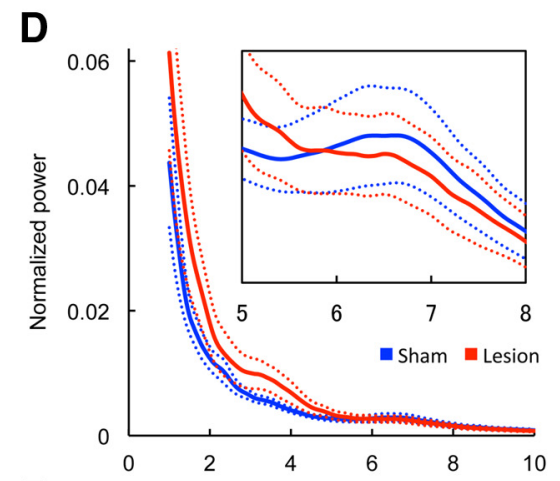

$\mathbf{F}$
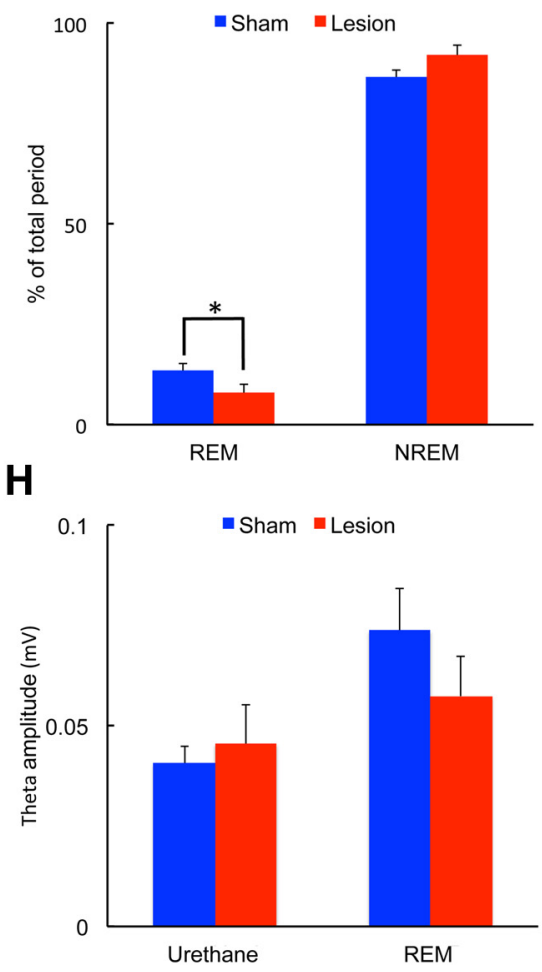

Figure 1. Intact lateral habenular function is essential for maintaining the hippocampal theta oscillation. $\boldsymbol{A}, \boldsymbol{B}$, Coronal sections of the habenula in rats that previously received either a sham $(\boldsymbol{A})$ or lesion operation $(\boldsymbol{B})$ showing the extent of destruction and gliosis ( $\boldsymbol{B}$, arrowheads). Asterisks indicate the medial habenula. Insets are magnified views of the gray-boxed areas in the left habenula. $\boldsymbol{C}, \boldsymbol{D}$, Power spectral density of LFP recorded from the pyramidal cell layer in the hippocampal CA1 of the sham-operated (blue line) and lesion-operated rats (red line) under urethane anesthesia ( $C, N=6$ for sham operation group, and $N=11$ for lesioned group) and during REM sleep ( $D, N=6$ for sham operation group, and $N=7$ for lesioned group). Solid and dotted lines indicate the mean and mean \pm SEM for the sham- and lesion-operated rats, respectively. Insets of each panel show enlarged views of the power spectrum for the theta wave under urethane anesthesia (3-6 Hz) and during REM sleep ( $5-8 \mathrm{~Hz})$. E, F, Bar graphs
For the neurotoxic lesion study, reduced expression of serotonin in the median raphe was confirmed by staining of the coronal sections with rabbit anti-serotonin polyclonal antibody (1:1000, Immunostar). The signal was visualized with peroxidase-conjugated anti-rabbit IgG and DAB-Ni.

\section{Retrograde labeling experiments}

The rat was anesthetized with $2 \%$ isoflurane and placed in the stereotactic apparatus (SR-8N). Tiny holes were made in the skull and dura mater, and a glass electrode containing either $1-2 \%$ Fluoro-Gold (Fluorochrome) in $10 \mathrm{~mm}$ cacodylate buffer or $0.5 \%$ cholera toxin subunit B-Alexa Fluor 488 conjugate (CTB488, Invitrogen) in $0.1 \mathrm{M} \mathrm{PB}$ was stereotactically positioned at the same coordinates used for the unanesthetized recording. Retrograde tracers were applied iontophoretically with $5 \mu \mathrm{A}$ current for $7 \mathrm{~min}$ for Fluoro-Gold ( $5 \mathrm{~s}$ on $/ 5 \mathrm{~s}$ off) and for $30 \mathrm{~min}$ for CTB488 (0.5 s on/0.5 $s$ off). For visualization of the tracers and the immunoreactivity against ChAT, rabbit antiFluoro-Gold (1:2000; AB153, Millipore), rabbit anti-Alexa Fluor 488 (1:2000; A11094, Invitrogen), and goat anti-choline acetyltransferase (1:500, AB144, Millipore) were used. Signals were visualized with secondary antibodies conjugated with fluorescent dyes (donkey anti-rabbit IgG-Alexa Fluor 488, A-21206, Invitrogen, 1:200; donkey anti-goat IgG-Alexa Fluor 594, A-11055, Invitrogen, 1:200) or secondary antibodies conjugated with horseradish peroxidase (1:200; peroxidase goat anti-rabbit IgG antibody, PI-1000, Vector Laboratories) and DAB-Ni as above. For colocalization of the Fluoro-Gold and Gad67 mRNA, sections were first processed for in situ hybridization and then stained with anti-Fluoro-Gold antibody as above.

\section{In situ hybridization}

In situ hybridization was performed as described previously (Amo et al., 2010). RNA probes for murine Gad67 mRNA were used for the hybridization reaction at $55^{\circ} \mathrm{C}$. For probe synthesis, the fragment containing nucleotide 7-625 of murine Gad67 mRNA (GenBank accession number NM_008077.4) was PCR amplified using mouse genomic DNA as a template. The amplified fragment was sub-

$\leftarrow$

showing the proportion of time with and without the hippocampal theta under urethane anesthesia (theta and nontheta periods, respectively, in $\boldsymbol{E}$ ) and with REM and NREM sleep $(\boldsymbol{F})$ in the animals subjected to the sham operation (blue, $N=$ 6), habenula-lesion (red, $N=11$ ), and habenula-lesion plus DHT injection into the median raphe (light blue, $N=7$ ). $\mathbf{G}, \boldsymbol{H}$, Bar graphs showing the mean duration of a single theta bout $(\boldsymbol{G})$ and theta amplitude $(\boldsymbol{H})$ of the theta oscillation in the hippocampus of the sham-operated (blue) and lesion-operated (red) rats under urethane anesthesia (bars on the left) and during REM sleep (bars on the right). ${ }^{*} p<0.05,{ }^{* *} p<0.01$, comparison with statistical significance (Scheffé's multiplecomparison test following one-way ANOVA for the threegroup data and one-tailed $t$ test for the two-group data). Values are presented as the mean \pm SEM. Scale bar, $1 \mathrm{~mm}$. 
A

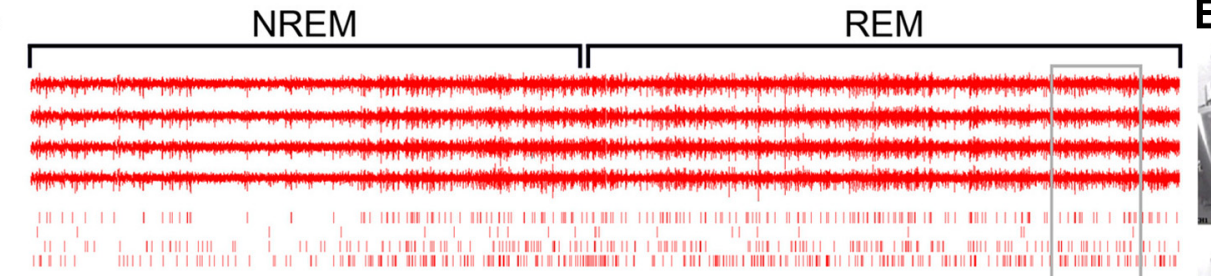

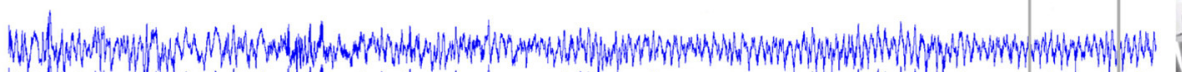

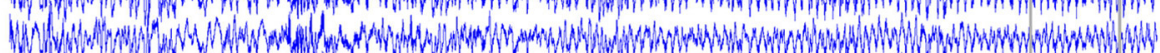

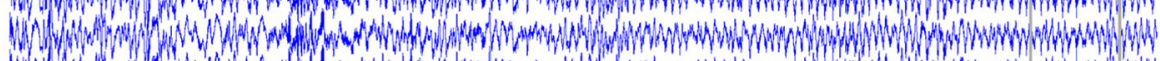
160.

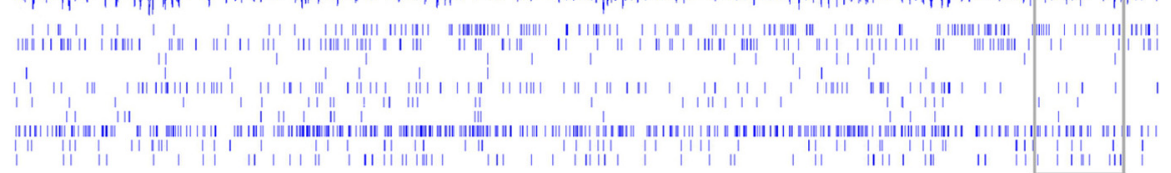

C
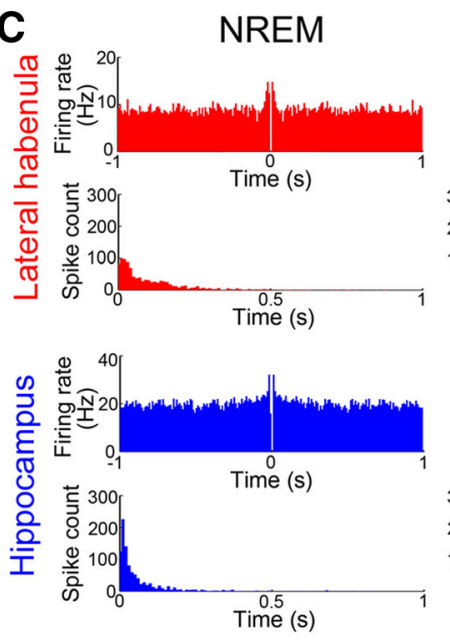

B
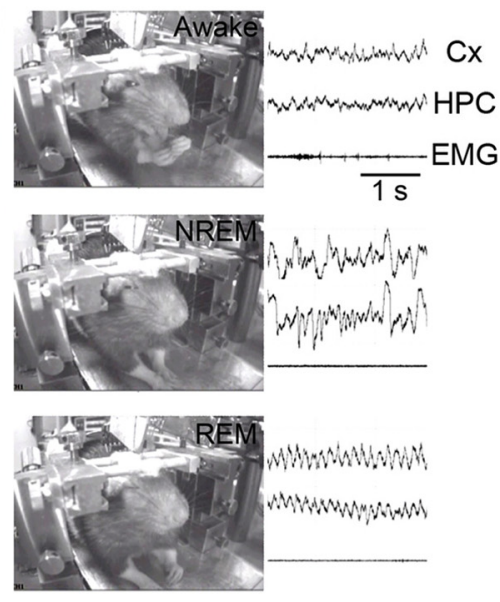
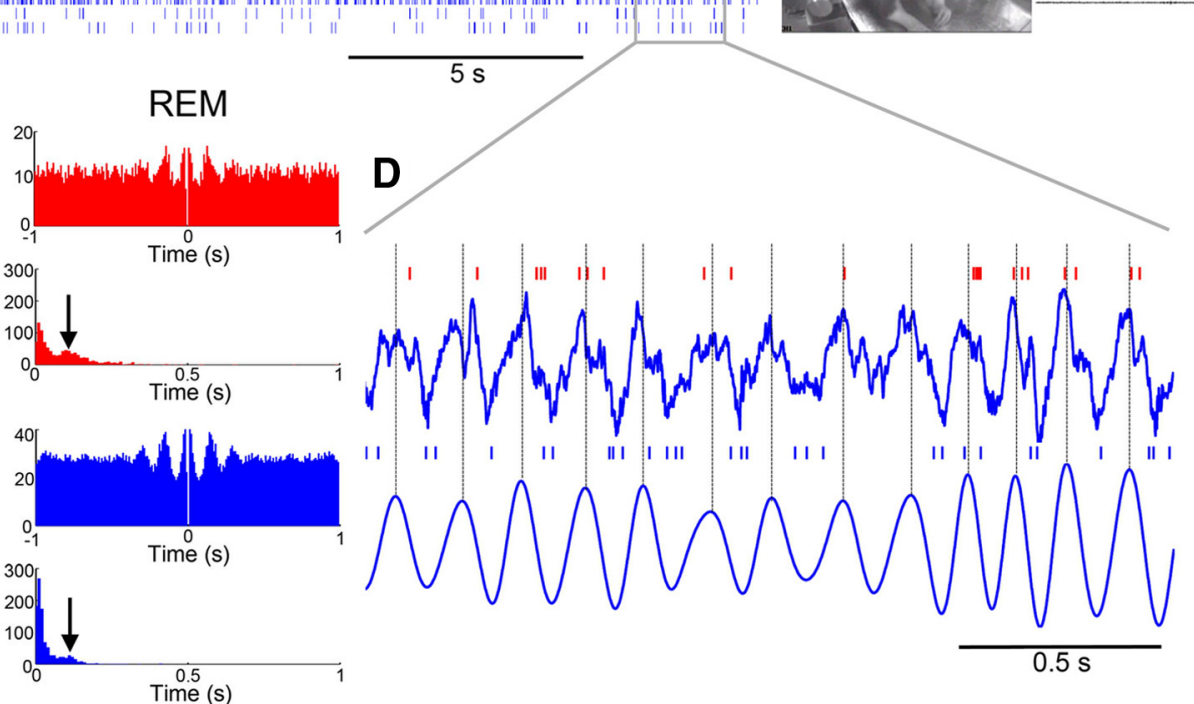

Figure 2. Changes in the firing pattern of the lateral habenular and hippocampal neurons according to the transition from NREM to REM sleep. $\boldsymbol{A}$, Raw data (upper four traces) and the extracted spike trains (raster below the raw traces) simultaneously recorded in the lateral habenula (red) and the pyramidal cell layer of the hippocampal CA1 region (blue) during non-REM and REM sleep. Top panel is rostral. $\boldsymbol{B}$, Representative recording of the brain and muscle activities in the awake (top), non-REM (middle), and REM sleep states (bottom). Panels represent the front view of the animals used for the video monitoring of behaviors (left), cortical electroencephalogram ( $\mathrm{Cx}$, top trace on the right), local field potential in the hippocampus (HPC, middle trace on the right), and EMG recorded from the neck muscle (bottom trace on the right). C, Autocorrelogram (top panels for each color) and interspike interval histogram (bottom panels) of the representative neurons in the lateral habenula (red) and hippocampus (blue) showing the changes in firing pattern according to the transition from NREM (left column) to REM sleep (right column). D, Raw (upper blue trace) and filtered (lower blue trace) theta traces and the spike train of a lateral habenular neuron (upper red raster) and a hippocampal neuron (lower blue raster) showing the preferential firing near peak and trough of each theta cycle, respectively, during the period indicated by the boxed area in $\boldsymbol{A}$.

cloned into the pBluescriptII vector (Stratagene), and the recombinant plasmid was used as the template to synthesize the antisense cRNA probes (digoxigenin and fluorescein labeling kit, Roche).

\section{Data analysis}

Determination of the sleep stages in unanesthetized animals. Behavioral states of the animals were classified into awake, REM sleep, and nonREM sleep (NREM) according to the procedure described previously (Costa-Miserachs et al., 2003) with modification and checking of video images for further confirmation of the correct classification of each stage. Briefly, the original hippocampal data were low-pass filtered at $500 \mathrm{~Hz}$ and down-sampled at $1000 \mathrm{~Hz}$. The multitaper method was used to window LFPs, and the fast Fourier transform was used to calculate spectral power for each $4 \mathrm{~s}$ epoch. We classified these epochs into awake, REM, or NREM, according to the mean and SD of delta $(0.5-4 \mathrm{~Hz})$ and theta power $(4-10 \mathrm{~Hz})$ and EMG values: periods with high EMG values and an intermediate theta/delta ratio were classified as awake; periods with high delta waves, low theta/delta ratio, and low EMG values were classified as NREM sleep; and periods with very high theta/delta ratio and very low EMG values were classified as REM sleep. Slow waves observed on cortical EEG or LFP were also used as auxiliary information for confirming the NREM sleep stage.

Determination of theta and non-theta periods in anesthetized animals. Data from the rat anesthetized with urethane was classified into theta, non-theta, and unclassified periods according to the spectral power of the hippocampal LFP. Theta epochs were detected by calculating the theta $(3-6 \mathrm{~Hz})$ to delta $(0.5-3 \mathrm{~Hz})$ frequency power ratio in $4 \mathrm{~s}$ windows of the LFP. A ratio greater than four in at least six consecutive windows marked theta periods, and a ratio less than two in at least six consecutive windows indicated epochs that are called non-theta periods (Csicsvari et al., 1999). We defined the unclassified period as when a theta/delta ratio matched neither the theta nor non-theta period, and we did not use these periods for further analysis.

Ripple detection. Ripple oscillations in the hippocampus were detected by the same methods as described previously with modification (Klausberger et al., 2003). Briefly, the hippocampal LFP was filtered at 150-250 $\mathrm{Hz}$ for the detection of ripples, and the power (root mean square amplitude) of the filtered signal was calculated in $10 \mathrm{~ms}$ windows. The threshold for ripple detection was set to $7 \mathrm{SDs}$ above the mean power. 
A
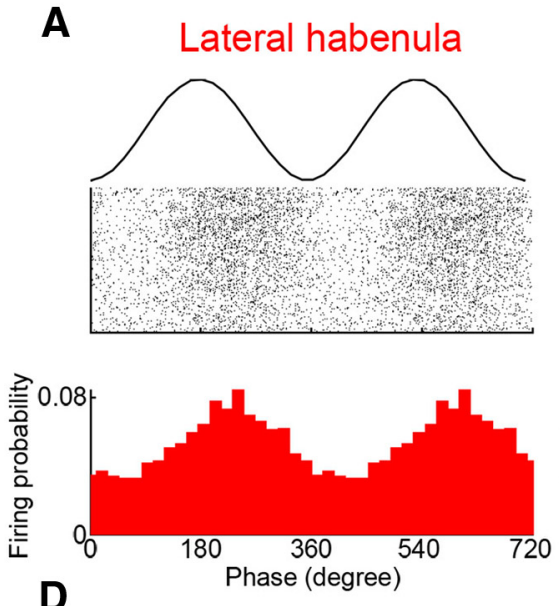

D

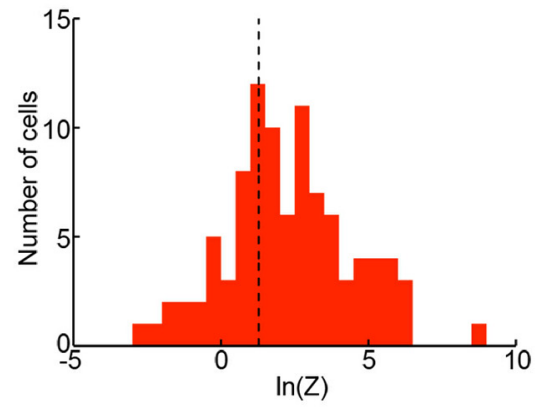

G

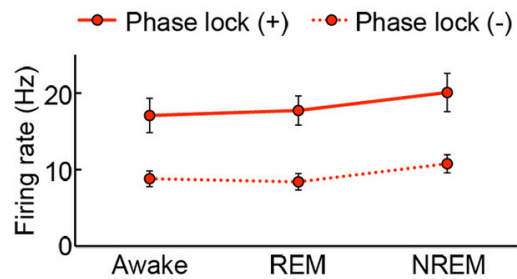

J

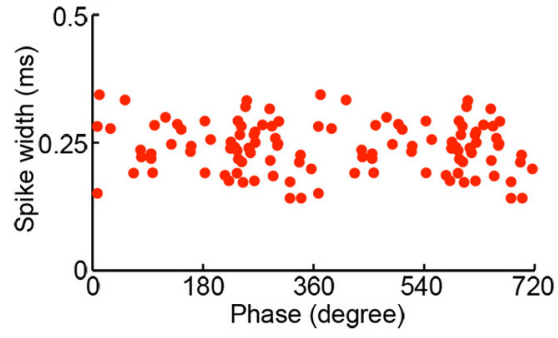

M

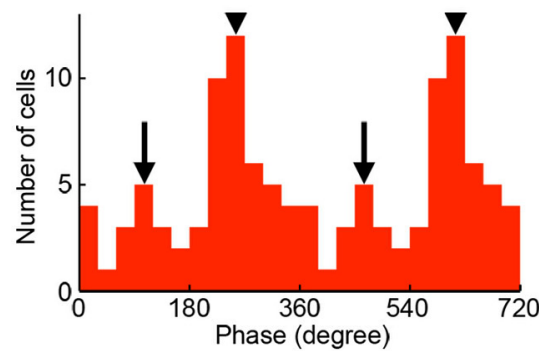

B
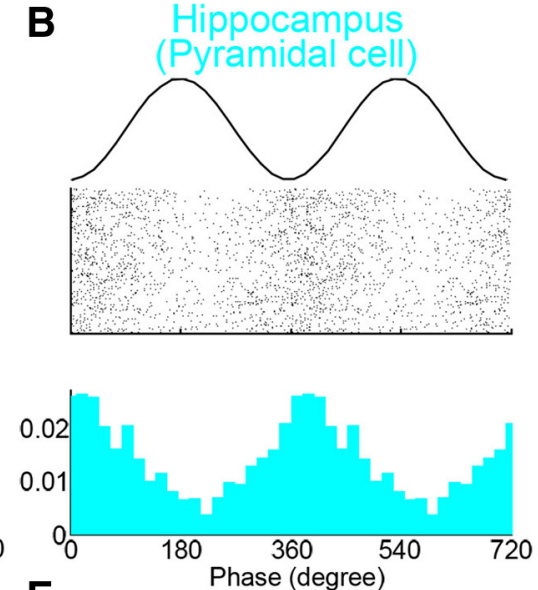

E

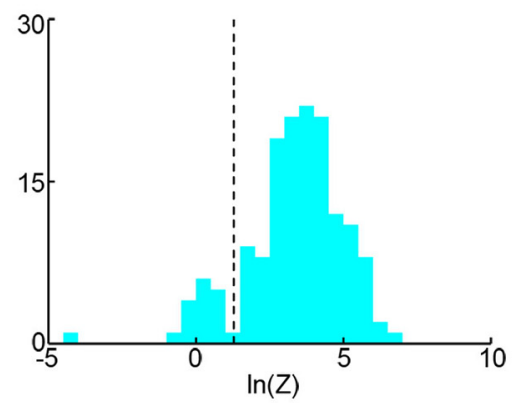

H

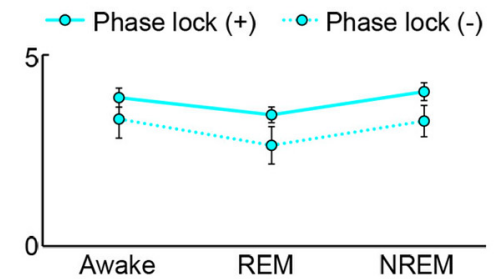

K

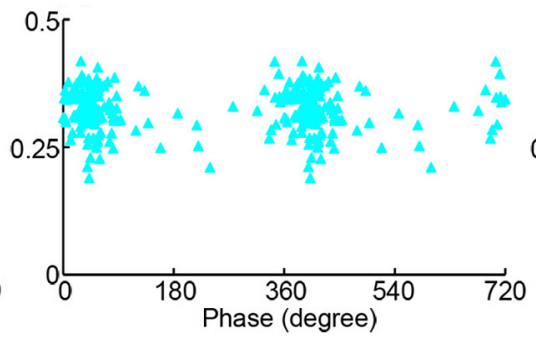

N

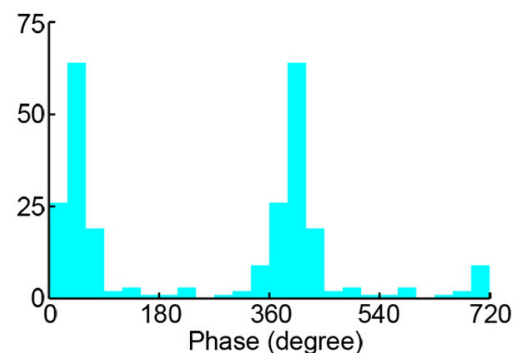

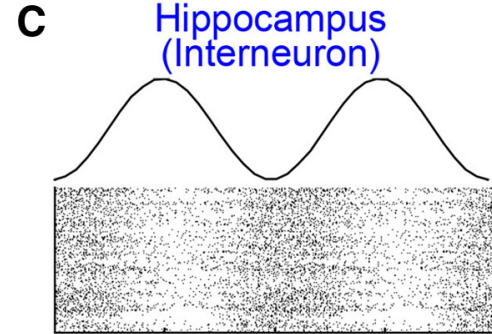

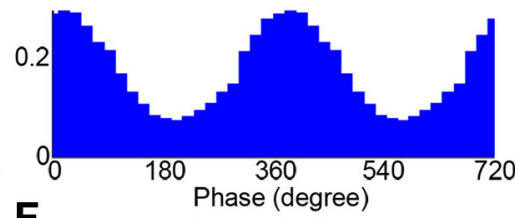

F

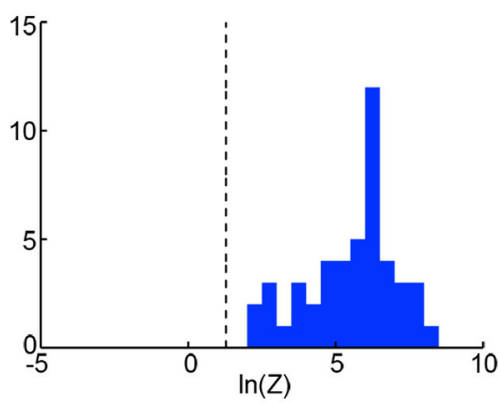

I

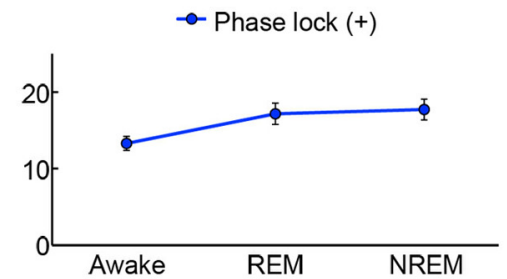

L

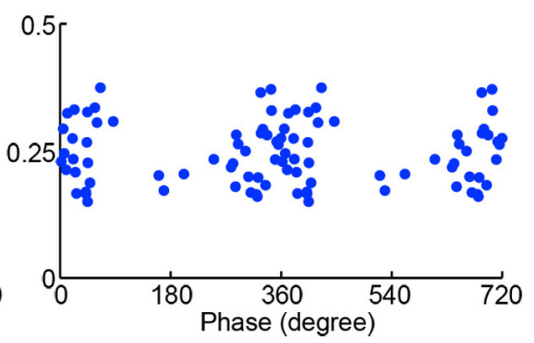

0

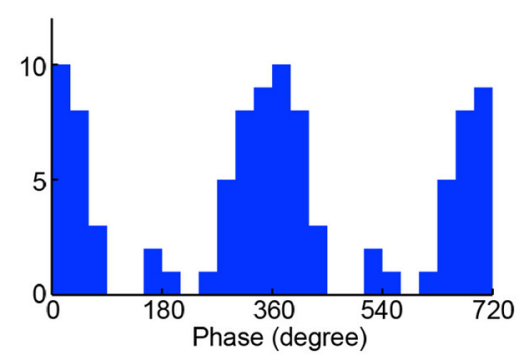

Figure 3. Phase-locking activities of the lateral habenular and hippocampal neurons. $\boldsymbol{A}-\boldsymbol{C}$, Examples of unit activity of neurons in the lateral habenula (red, $\boldsymbol{A}$ ) and a pyramidal cell (light blue, $\boldsymbol{B}$ ) and an interneuron in the hippocampus (dark blue, $C$ ) showing in the raster plots (top panels) and in the phase histogram of the firing probability within the time window made by subdividing each theta cycle $\left(360^{\circ}\right)$ into 20 bins (bottom panels). Data were replicated over two theta cycles for visual clarity. D-F, Distribution of log-transformed Rayleigh's $Z$ value, a statistical value used to evaluate the significance of phase locking for the lateral habenular neurons $(\boldsymbol{D})$, the pyramidal cells $(\boldsymbol{E})$, and interneurons $(\boldsymbol{F})$ in the hippocampus. Dashed black (Figure legend continues.) 
Spike sorting. Multiunit and juxtacellular recording data were processed to isolate spike events by the automatic spike-sorting program, combining principal component analysis (12 feature dimensions; highpass filter, $300 \mathrm{~Hz}$; time resolution, $20 \mathrm{kHz}$; spike-detection interval, $>0.5 \mathrm{~ms}$ ) with robust variational Bayes for spike clustering (Takekawa et al., 2010). Next, the sorted spike clusters were combined, divided, and discarded manually to refine single-neuron clusters using Klusters and NeuroScope (Hazan et al., 2006).

Phase-locking detection and characterization. Phase in theta oscillation was defined by the zero crossing method (Siapas et al., 2005). Briefly, the special points were the negative to positive and positive to negative zero crossings of the filtered hippocampal LFP $(4-10 \mathrm{~Hz})$, and they were assigned instantaneous phases of $-90^{\circ}$ and $90^{\circ}$, respectively. The presence of phase locking was evaluated by applying the Rayleigh test for circular uniformity on distribution of the firing probability along the theta phase value. For statistical analysis, we used circStat MATLAB scripts to calculate mean direction (preferred phase) and Rayleigh's $Z$ value (Berens, 2009). The approximation $p=e^{-Z}$ was adopted in all cases, since the number of samples exceeded 50 (Fisher, 1993).

Data analyses, except for spike sorting and circular analysis, were conducted by using custom-made scripts and scripts available from the laboratory of Gyorgy Buzsáki at Rutgers University (Newark, NJ) for MATLAB (MathWorks).

\section{Results}

\section{Lateral habenula is indispensable for maintaining the duration of the hippocampal theta oscillation during REM sleep}

To address whether intact activity in LHb plays an essential role in modulating the hippocampal theta oscillation, we destroyed the rat LHb by introducing an electrolytic lesion and recorded the oscillatory activity in the hippocampus of the urethane-anesthetized rats in the stereotaxic frame (6 shamoperated and 11 lesioned rats) or of sleeping rats in the freely behaving preparation (6 sham-operated and 7 lesioned rats) (Fig. $1 A, B)$. The hippocampal theta oscillation was of a lower frequency in the animals under urethane anesthesia $(3-6 \mathrm{~Hz})$ than in those measured during REM sleep as reported previously (Kramis et al., 1975). In both conditions, rats with an LHb lesion (Fig. $1 C, D$, red lines) showed a significant reduction in power spectral density at the theta frequency throughout all recording periods, compared with the sham-operated rats (Fig. 1C,D, blue lines).

Reduction in the power spectral density at the theta frequency reflected a shortening of the total period showing theta oscillation (Fig. $1 E, F$ ) and, in particular, the duration of a single theta bout in the LFP of hippocampus (Fig. 1G) under anesthesia (Fig. $1 G$, left) and during REM sleep (Fig. $1 G$, right). These changes resulted in a reduced proportion of REM sleep periods among the total sleep time (Fig. 1F, left) without affecting the non-REM sleep period (Fig. $1 F$, right). Reduced periods with theta oscillation under anesthesia and during REM sleep could not be attributed to changes in the characteristics of each theta cycle, since the

\footnotetext{
(Figure legend continued.) lines indicate the $p=0.01$ significance thresholds, which were used to identify the phase-locking neurons. $\mathbf{G}-\mathbf{I}$, Changes in the mean firing rate of the recorded neurons showing phase locking (solid lines) and those without phase-locking activity (dotted lines) in the lateral habenula ( $\boldsymbol{G}$ ) and hippocampus (pyramidal cells in $\boldsymbol{H}$ and interneurons in $\boldsymbol{I}$ ) across the brain states. Error bars indicate SEMs. $J-L$, Scatter plots showing the preferred phase of the phase-locking neurons against the spike width in the lateral habenula $(\boldsymbol{J})$ and hippocampus (pyramidal cells in $\boldsymbol{K}$ and interneurons in $\boldsymbol{L}$ ). $\boldsymbol{M} \mathbf{- 0}$, Histograms showing the frequency of the theta phase preferred by the phase-locking neurons recorded in the lateral habenula $(\boldsymbol{M})$ and hippocampus (pyramidal cells in $\boldsymbol{N}$ and interneurons in $\mathbf{0}$ ). Arrows and arrowheads in $\boldsymbol{M}$ indicate the neuronal groups in the lateral habenula preferring the ascending and descending phases of the theta cycle, respectively.
}

$\leftarrow$ amplitude (Fig. $1 H$ ) and frequency (data not shown) of theta oscillation were comparable between sham-operated and lesioned rats. General impairment of the neuronal excitability in hippocampus also could not account for the reduced theta power during the total recording period, since we did not observe significant changes in the frequency of other oscillatory events during sleep such as ripple oscillation (mean \pm SEM, $0.17 \pm 0.05 \mathrm{~Hz}$ for sham; $0.13 \pm 0.01 \mathrm{~Hz}$ for lesion group; $p=0.27$ with onetailed $t$ test).

Previous studies identified many brain regions whose activation or inhibition modulates the theta oscillation in the hippocampus including the medial septum (MS)/diagonal band complex, supramammillary nucleus, and nucleus pontis oralis (Vertes, 2005). These findings suggested that the net effect of these region-specific positive and negative modulations regulate the hippocampal theta oscillation. A considerable body of evidence indicates that the serotonin-containing median raphe suppresses the theta and desynchronizes local field potential in the hippocampus (Vertes, 2005). Indeed, theta oscillation occurred in the rat hippocampus more frequently when the serotonergic neurons in the raphe were lesioned with a neurotoxin, 5,7-DHT (Vanderwolf et al., 1989).

Since the lateral habenula specifically and directly projects to the median raphe, we questioned whether LHb needs activity in the serotonergic raphe to modulate theta oscillation in the hippocampus. To address this possibility, we examined the hippocampal theta oscillation of the LHb-lesioned rats in which the serotonergic neurons in the median raphe were destroyed by 5,7DHT. We observed a significant reduction in the number of serotonergic neurons in the median raphe 1 week after the 5,7DHT injection by serotonin immunohistochemistry (data not shown). In addition, with reduced serotonergic activity following the injection of 5,7-DHT, an LHb lesion could not affect the total duration of theta oscillation (Fig. 1E, light blue) or the duration of a single theta bout in the LFP of hippocampus (Fig. $1 G$, light blue). This implies that the effect of LHb lesions on theta maintenance depends on the integrity of the serotonergic median raphe.

These findings suggested that LHb plays a critical role in maintaining the duration of theta oscillation in the hippocampus both under anesthesia and during sleep via the serotonergic median raphe.

\section{Brain state-specific firing of the lateral habenular neurons with phase-locking activity}

To determine when and how the LHb neurons are activated, we simultaneously recorded LFP and single-unit activity in the CA1 pyramidal cell layer of the HPC $(n=199)$ together with a singleunit activity in $\mathrm{LHb}$ ( $n=95$ neurons) in the rats $(N=25$ rats; Fig. $2 A$ ). We targeted these two regions using the stereotactic recording apparatus with which the head-restrained rats were habituated to show awake, REM, or NREM sleep states (Fig. 2B).

Since the hippocampal neurons show a diverse morphology and firing pattern dependent upon the neuronal subtypes (Klausberger and Somogyi, 2008), the unit activities from HPC were classified into those with the putative pyramidal cell (152/199; hereafter called "pyramidal cell") and putative interneuron (47/ 199; hereafter called "interneuron") according to the spike shape and firing pattern, as described previously (Csicsvari et al., 1999). The firing pattern of $\mathrm{LHb}$ neurons appeared tonically active, as observed previously (Zhao and Rusak, 2005; Matsumoto and Hikosaka, 2007), and the mean ( \pm SEM) firing rate of LHb neurons was $14.3 \pm 1.3 \mathrm{~Hz}$. Among these activities, we observed that 
A

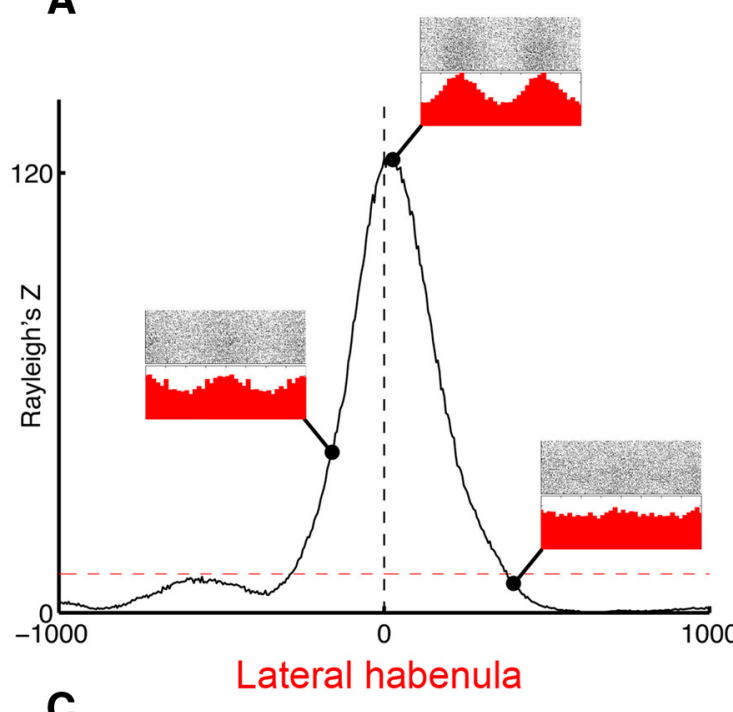

C

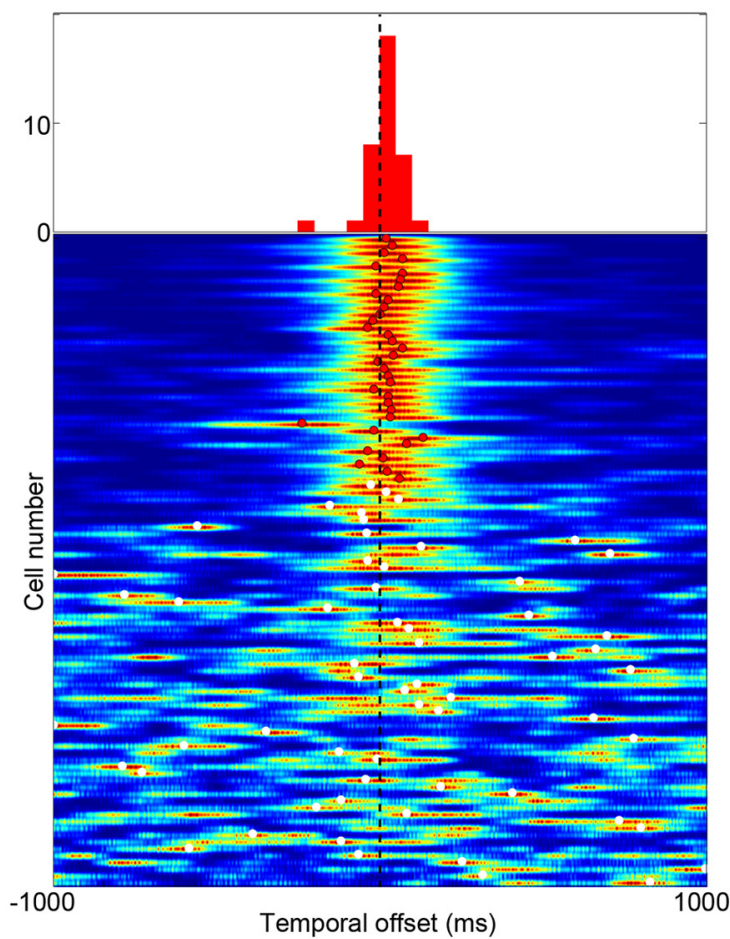

E

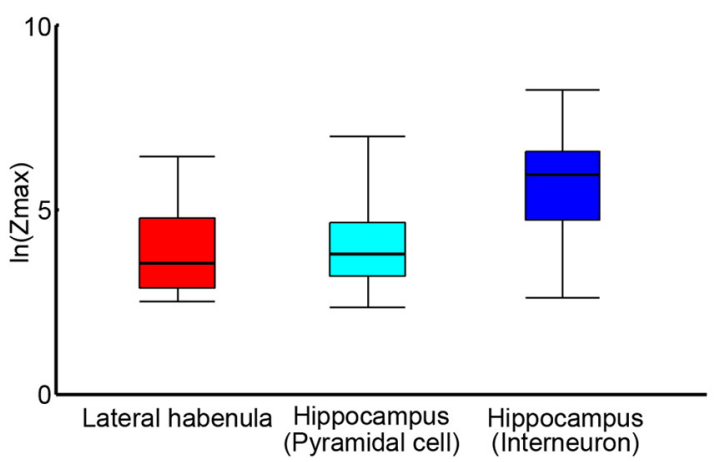

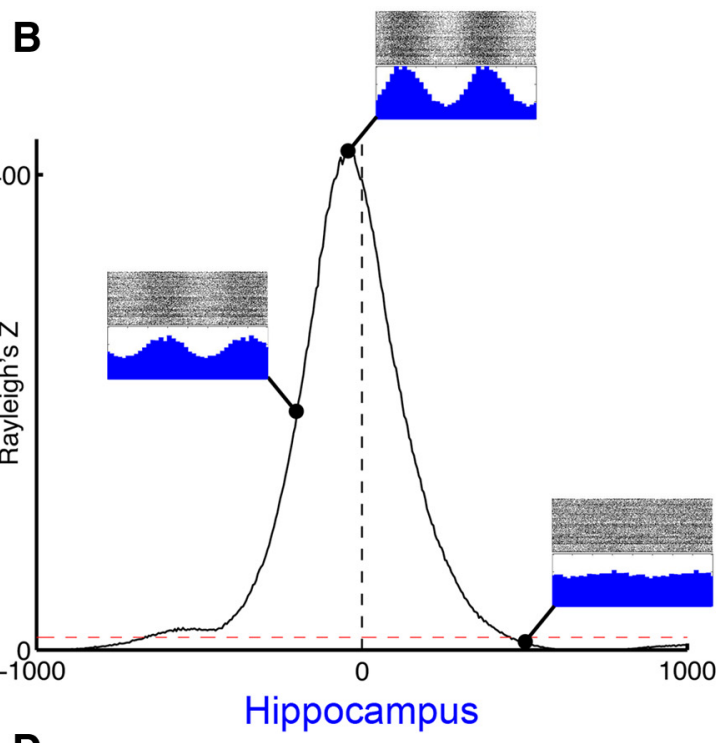

D

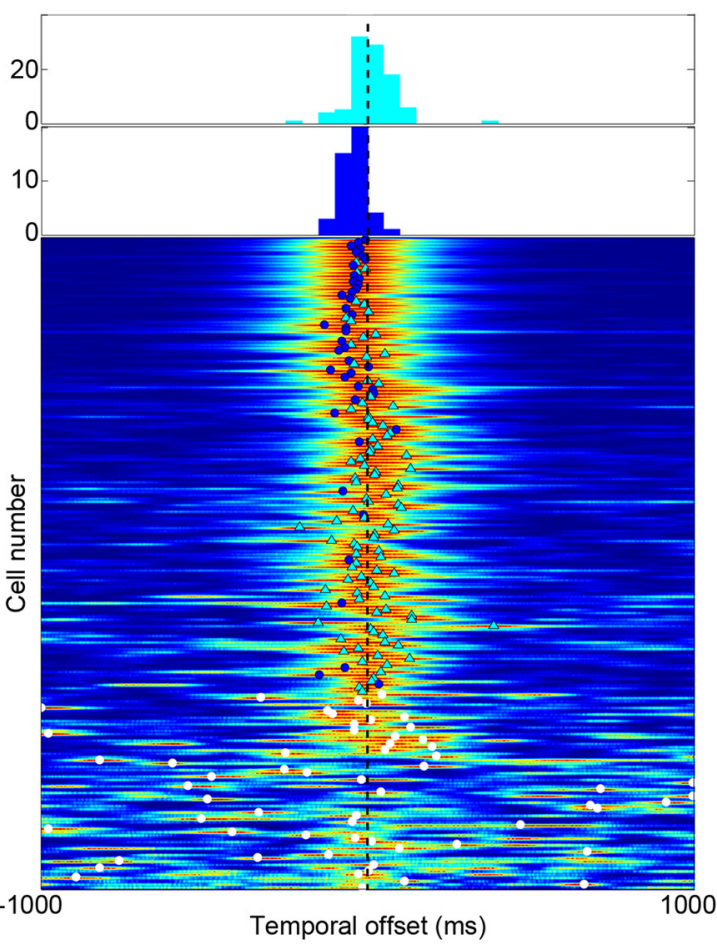

$\mathbf{F}$

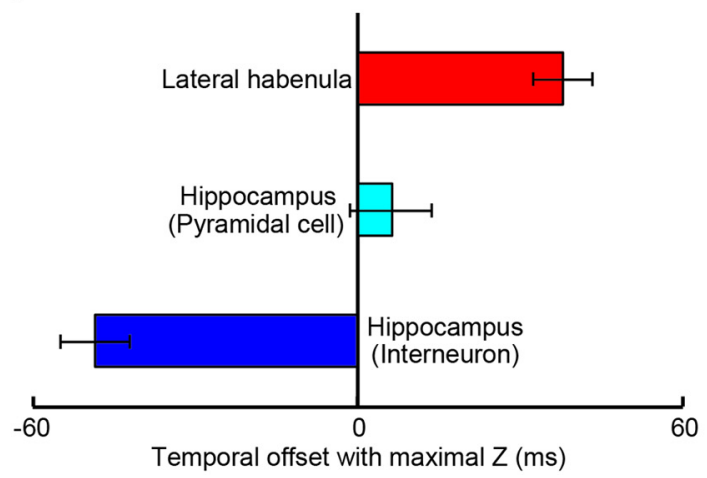

Figure 4. Coordination of phase-locking activity in the lateral habenula with fluctuating cycles in the hippocampal theta oscillation. $A, B$, Rayleigh's $Z$ value computed as a function of temporal offset (from - 1000 to $1000 \mathrm{~ms}$ with $5 \mathrm{~ms}$ bins) between the hippocampal theta trace and the spike train of lateral habenular $(\boldsymbol{A})$ and hippocampal ( $\boldsymbol{B}$ ) neurons (positive temporal offset indicates shifting the theta trace forward). The inserts explicitly show the theta-triggered rasters and firing probability along the binned phase value at temporal (Figure legend continues.) 
some LHb neurons changed firing patterns when the animals came to be in REM sleep and showed oscillatory firing patterns in the theta range $(4-10 \mathrm{~Hz})$ as detected by novel positive peaks at $\pm 120-150 \mathrm{~ms}$ in autocorrelograms and interspike interval histograms (Fig. 2C). This change in the firing pattern was maintained during a single REM sleep episode, which lasted $>3$ min and was consistently observed throughout recording in all cases. This finding indicated that the pattern of $\mathrm{LHb}$ neuronal firing became oscillatory and synchronized during REM sleep.

\section{Close temporal association of the neuronal activities in the lateral habenula with the hippocampal oscillation}

Appearance of the hippocampal theta characterizes REM sleep (Jouvet, 1969) and is believed to be critical for temporal coding and decoding of active neuronal ensembles and the modification of synaptic efficacy (Buzsáki, 2002). Since the oscillatory activity that we observed in LHb neurons was in the theta frequency range, we suspected that changes in firing patterns of the $\mathrm{LHb}$ neuron during REM sleep could have a close temporal association with the hippocampal theta oscillation. Indeed, in a closer view, we found that some LHb neurons with oscillatory activity appeared to fire preferentially around the positive peak of the hippocampal theta at a glance (Fig. $2 D$, red raster), which was in clear contrast to the oscillatory firing of the hippocampal neurons at a trough of the theta, as reported previously (Csicsvari et al., 1999) [Fig. 2C,D (in $D$, ark blue raster)].

To determine the significance of this phase-locking firing pattern quantitatively, we analyzed the firing pattern of each neuron by phase histogram to show the probability of the neuronal firing observed in each theta cycle whose length fluctuated time after time during REM sleep (Fig. $3 A-C$ ). Then, we checked the statistical significance of the phase-locking activity of the LHb neurons by Raleigh's $Z$ value, as used for the analysis of circular data (Fisher, 1993; Siapas et al., 2005). Using this measure, the neurons showing a more significant peak in the phase histogram were characterized by a higher Rayleigh's $Z$ value (Siapas et al., 2005). According to this analysis, we identified the LHb neurons showing a significant peak in the firing probability for each cycle of the hippocampal theta oscillation during REM sleep (Fig. $3 A$ ), as observed in the pyramidal cells and interneuron in HPC (Fig. $3 B, C)(p<0.01)$. This analysis revealed that $61.1 \%$ of the recorded LHb neurons (58 of 95 neurons) showed phase-locking activity, as observed in the hippocampus where $88.2 \%$ of the

$\leftarrow$

(Figure legend continued.) offsets $=-200,25$, and $400 \mathrm{~ms}$ for $A$, and $-250,-45$, and $500 \mathrm{~ms}$ for $\boldsymbol{B}$. Vertical black dashed lines and horizontal red dashed lines indicate zero in temporal offset and the threshold of $Z$ value for the statistical significance $(p=0.01 / 201$ by Bonferroni's correction), respectively. $C, D$, Pseudocolor plots of the populational Z-shift analysis summarizing the Rayleigh's $Z$ value normalized with the maximal $Z$ value as a function of temporal offset for each neuron. $Z$ values for the dataset shifted by $5 \mathrm{~ms}$ for each neuron were displayed in rows and were sorted by maximal $Z$ value in descending order $(n=95$ for the lateral habenula and $n=199$ for the hippocampus). Red, light blue, and dark blue dots indicate the recordings from the lateral habenular neurons, putative hippocampal pyramidal cells, and putative hippocampal interneurons, respectively. Dots (lateral habenular and putative hippocampal pyramidal neurons) and triangles (putative hippocampal interneurons) indicate the location of the temporal offset at which the distribution showed the maximal $Z$ value, and the color signifies whether (red, lateral habenular neurons; light blue, putative pyramidal cells; dark blue, putative interneurons) or not (white) the maximal $Z$ value exceeded the significance threshold. $E$, Box plots showing the log-transformed value of the maximal $Z$ value for the lateral habenular neurons (red), pyramidal cells (light blue), and interneurons (dark blue) in the hippocampus. $\boldsymbol{F}$, Bar graphs showing the mean values of the temporal offset with maximal $Z$ value for the lateral habenular neurons (red), hippocampal pyramidal cells (light blue), and interneurons (dark blue). Error bars indicate SEM. pyramidal cells (134 of 152 neurons) and all interneurons (47 neurons) showed phase-locking activity (Fig. $3 D-F$ ).

Since hippocampal theta oscillation was predominantly observed during REM sleep, when the neuron firing rate starts to decrease in particular brain regions such as locus ceruleus (Hobson et al., 1975), it is possible that LHb neurons show constitutive phase-locking activity with increased firing rate only during REM sleep. To address this possibility, we examined the firing rate changes of LHb neurons during awake, REM, and NREM sleep. Population analysis revealed that neither of the two groups of $\mathrm{LHb}$ neurons, with and without phase-locking activity, showed significant changes in firing rate across the brain states (Fig. 3G-I). This observation indicated that the phase-locking activity of $\mathrm{LHb}$ neurons could not simply be accounted for by intrinsic firing rate changes in REM sleep. Instead, it suggested that phase-locking activity in a subpopulation of LHb neurons might be under the influence of the incoming theta-related neural activity. Intriguingly, the analysis also revealed that $\mathrm{LHb}$ neurons and pyramidal cells in HPC showing phase-locking activity persistently showed higher mean firing rates compared with those without that activity, regardless of the brain states (Fig. $3 G-I$ ).

\section{Segregation in preferred theta phase between the lateral habenular and the hippocampal neurons}

Next, we checked the timing within each theta cycle at which the LHb neurons are activated by calculating the mean preferred phase of each neuron that showed phase-locking activity. Pyramidal cells and most of the interneurons preferred the trough of the theta cycle (Fig. $3 K, L, N, O$ ), although a small number of interneurons preferred the peak (Fig. 3O, arrows) as reported (Csicsvari et al., 1999; Klausberger et al., 2003). In contrast, LHb neurons avoided firing at the trough of the theta cycle (Fig. $3 J, M)$. Intriguingly, LHb neurons were further subdivided into two groups according to the difference in preferred phase: neurons that peak at the ascending phase of the theta cycle and those peaking at the descending phase (Fig. $3 M$, arrows and arrowheads, respectively).

Thus, this analysis revealed that LHb neurons tend to cease firing at the negative peak of the hippocampal theta rhythm, where HPC neurons preferentially fire, and that two populations of LHb neurons fired at distinct phases within each cycle of the hippocampal theta oscillation.

\section{Phase-locking activity in the lateral habenular neurons depends on fluctuations in the hippocampal theta rhythm} Despite theta oscillation in the hippocampus playing a critical role in the efficient encoding of spatial and episodic memory, oscillatory activity in theta frequency is not specific to the hippocampus. For example, this phase-locking phenomenon alone cannot provide conclusive evidence to say that LHb works in accordance with the hippocampal theta, since it is still possible that the intrinsic rhythmicity of LHb at a range of $4-10 \mathrm{~Hz}$, but independent of the hippocampal theta, can induce the apparent phase locking (Siapas et al., 2005). Furthermore, a significantly phase-locked neuron may not necessarily reveal itself to be rhythmic, as defined by autocorrelation or interspike interval measures, and the spike train must coordinate with the temporal fluctuation in hippocampal theta oscillation if the LHb encodes the temporal information for maintaining the hippocampal theta oscillation.

To check whether the observed phase locking is not simply due to intrinsic rhythmicity of the neuronal firing at $4-10 \mathrm{~Hz}$, but rather that it explicitly depends on coordination of the LHb spike 


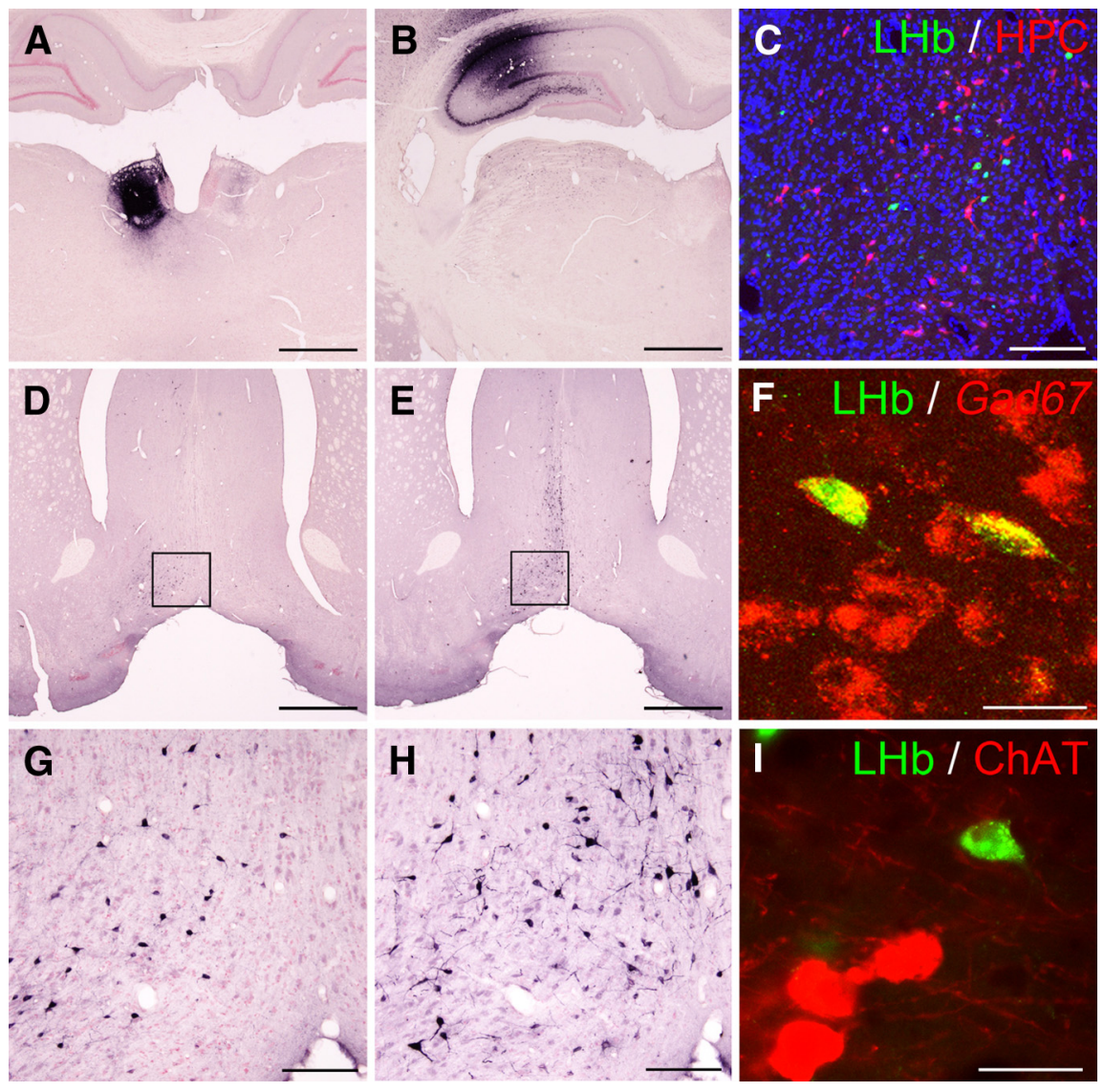

Figure 5. Diagonal band of Broca as a common input to the lateral habenula and hippocampus. $\boldsymbol{A}, \boldsymbol{B}, \boldsymbol{D}, \boldsymbol{E}, \boldsymbol{G}, \boldsymbol{H}$, Coronal sections of the lateral habenula $(\boldsymbol{A})$, hippocampus $(\boldsymbol{B})$, and diagonal band $(\boldsymbol{D}, \boldsymbol{E}, \boldsymbol{G}, \boldsymbol{H})$ showing the localization of retrograde tracers injected into the lateral habenula (cholera toxin B conjugated with Alexa Fluor 488, black in $A$ ) and hippocampus CA1 region (Fluoro-Gold, black in $\boldsymbol{B})$, and the distribution of retrogradely labeled neurons $(\boldsymbol{D}, \boldsymbol{E}, \boldsymbol{G}, \boldsymbol{H}) . \boldsymbol{G}$ and $\boldsymbol{H}$ are magnified views of the boxed areas in $\boldsymbol{D}$ and $\boldsymbol{E}$, respectively. $\boldsymbol{C}$, A coronal section of the vertical limb of the diagonal band of Broca showing the retrogradely labeled neurons projecting to the lateral habenula (cholera toxin B conjugated with Alexa Fluor 488, green) and to the hippocampus (Fluoro-Gold, red). Note that both the red and green signals were distributed in the vDBB. Sections were counterstained with DAPI (blue). $\boldsymbol{F}, \boldsymbol{I}$, Representative images of the VDBB neurons projecting to the lateral habenula showing colocalization of the tracer (green) with Gad67 mRNA (red in $\boldsymbol{F}$ ) and ChAT (red in I). Scale bars: $\boldsymbol{A}, \boldsymbol{B}, \boldsymbol{D}, \boldsymbol{E}, 1 \mathrm{~mm} ; \boldsymbol{C}, \boldsymbol{G}, \boldsymbol{H}, 300 \mu \mathrm{m} ; \boldsymbol{F}, \boldsymbol{I}, 25 \mu \mathrm{m}$. locking activity with the theta oscillation than the others $(p<0.001$, Kruskal-Wallis test with multiple comparison with Scheffé's method) (Fig. 4E).

These results indicated that a subpopulation of $\mathrm{LHb}$ neurons fire in coordination with anatomically distant structures such as the hippocampus with phaselocking activity toward the fluctuating theta rhythm.

\section{Diagonal band of Broca as a common source of cholinergic and GABAergic input to the lateral habenula and hippocampus}

The emergence of the oscillatory activity in LHb that coincided with the hippocampal theta raised the possibility that the neuronal activity in LHb may be influenced and entrained by the outputs from the septo-hippocampal system under anesthesia and during REM sleep. Indeed, our Z-shift analysis further showed that the neuronal activities in LHb phase locked to the hippocampal theta maximally when theta was shifted toward past direction (mean $\pm \mathrm{SEM}, 37.9 \pm 5.5 \mathrm{~ms}$ ), while the neuronal activities in the hippocampal interneurons did when the theta was shifted toward opposite direction (interneurons, $-48.5 \pm 6.5 \mathrm{~ms}$ ) as reported previously (Siapas et al., 2005; Hangya et al., 2009) (Fig. 4F). This suggested that the LHb neuron might be under the influence of the hippocampal theta rhythm, which occurred earlier, and be entrained by the outputs from the septo-hippocampal system after some delays.

To identify the origin responsible for timing to the fluctuating hippocampal theta oscillation, $Z$-shift analysis was conducted in which we artificially shifted the LHb spike train with reference to the hippocampal theta with a temporal offset toward the past and future direction, and calculated Rayleigh's Z value to evaluate the statistical significance (Siapas et al., 2005). This analysis revealed that stronger phase locking was observed only around the zero shift, since increasing the temporal offset abruptly diminished the Rayleigh's $Z$ value both in LHb and HPC (Fig. 4A,B). This finding indicated dependency of the phase-locking activity on the ongoing fluctuation in HPC theta oscillation. Although the statistical evaluation of those values needs correction because of the multiple comparison across bins in Z-shift analysis, 37.9\% of all LHb neurons still showed statistical significance even after the statistical correction (36/95; Fig. $4 C$, filled red circles) as in $62.8 \%$ of all pyramidal cells $(125 / 199$; Fig. $4 D$, filled light blue circles) and $97.9 \%$ of all interneurons in HPC (46/47; Fig. 4D, filled dark blue circles). The remaining neurons had multiple peaks with lower values deviated from the zero offset or did not show a decrease in $Z$ value in proportion to the size of perturbation. Comparison of the maximal $Z$ value further showed that the degree of phase locking in LHb was comparable to that in the putative pyramidal cells in HPC, while the putative interneurons in HPC showed much stronger phase- entraining the phase-locking activity in LHb and HPC, we searched the brain regions that send the axons both to LHb and HPC by double-labeling experiments that differentially label the neuronal cell bodies retrogradely from the LHb and HPC (Fig. $5 A, B)$. The results corroborated the distribution of the neurons projecting to $\mathrm{LHb}$ and the hippocampus $\mathrm{CA} 1$ region in the subcortical structures such as the vertical limb of the DBB (vDBB), ventral pallidum, preoptic areas, entopeduncular nucleus, ventral tegmental area (VTA) and median raphe for LHb (data not shown) (Herkenham and Nauta, 1977), and the MS, DBB, nucleus reuniens thalami, ventral tegmental area, and median raphe for HPC (data not shown) (Wyss et al., 1979). Except for the region sending the projection to diverse targets in the brain as diffuse modulatory system, such as ventral tegmental area and median raphe, we found that $\mathrm{VDBB}$ is the region that sends the outputs to both LHb and HPC (Fig. 5D,E). Neurons labeled retrogradely from $\mathrm{LHb}$ and $\mathrm{HPC}$ were intermingled and distributed in the same area in vDBB (Fig. $5 G, H$ ) but were not colocalized (Fig. $5 C$ ). The majority of vDBB neurons projecting to $\mathrm{LHb}$ was GABAergic, since $85.3 \%$ of vDBB neurons projecting to $\mathrm{LHb}$ expressed Gad67 mRNA, a marker for GABAergic neurons (81/95 neurons), and $4.9 \%$ of neurons expressed choline acetyl- 
transferase, a marker for cholinergic neurons (17/346 neurons) (Fig. 5I).

Taking into consideration the facts that vDBB and MS have been known as pacemaker regions for generating the hippocampal theta (Green and Arduini, 1954; Petsche et al., 1962), and the neuronal activity in those regions leads the hippocampal theta rhythm (Hangya et al., 2009), our results suggest that GABAergic neurons in $\mathrm{vDBB}$ should be a primary candidate for entraining the phaselocking activity in LHb.

\section{Localization of the phase-locking neurons in the medial division of the lateral habenula}

Habenula has been implicated in diverse behaviors such as nociception, sexual behavior, and learning, and it was recently pointed out that the heterogeneity of the neural pathways in this tiny area may make it difficult to discern the physiological function of the habenular circuitry so far (Klemm, 2004). LHb can be divided into the medial (LHbM) and lateral divisions (LHbL), and were further subdivided into at least seven subnuclei recently according to the morphological characters (Andres et al., 1999; Geisler et al., 2003; Kowski et al., 2009; Aizawa et al., 2012). Thus, we suspected that LHb neurons showing phase-locking activity are not homogeneously distributed but rather concentrated in specific subnuclei with distinct neural connectivities relevant to the hippocampal theta generation.

To determine the accurate localization of the phase-locking neurons in the subnuclei of LHb, we adopted a juxtacellular labeling technique to label the single neurons with or without phase locking to the hippocampal theta in the anesthetized rats (Pinault, 1996). Among a total of 92 recorded neurons, 37 neurons were visualized successfully, and 21 of 37 neurons showed phase-locking activity as observed in REM sleep (Fig. 6A,B). All of the recorded neurons had multiple processes regardless of the localization in the subnuclei (Wilcox et al., 1988; Kim and Chang, 2005) (Fig. 6C). Recorded neurons were distributed throughout $\mathrm{LHb}$ subnuclei along the mediolateral axis (Fig. 6D,E). We did not see any difference in firing rate, spike amplitude, and spike width between LHbM and LHbL (Fig. 6E; $p=0.69$, 0.23 , and 0.17 , respectively; Mann-Whitney $U$ test). Intriguingly, Rayleigh analysis revealed that the proportion of phase-locking neurons varied according to the subnuclei [e.g., 9 of 11 in the central part of $\mathrm{LHbM}$ (LHbMC), but 6 of 17 in the magnocellular part of LHbL (LHbLMc)], and LHb neurons with phase-locking activity local-

A

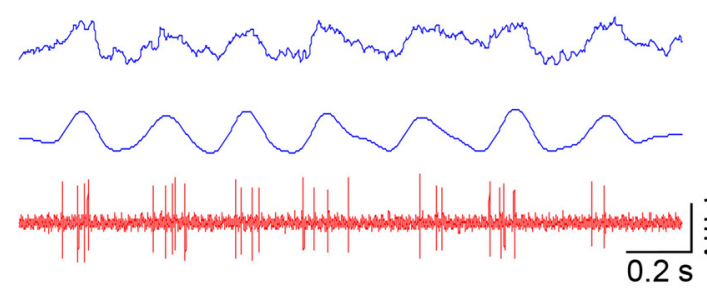

B
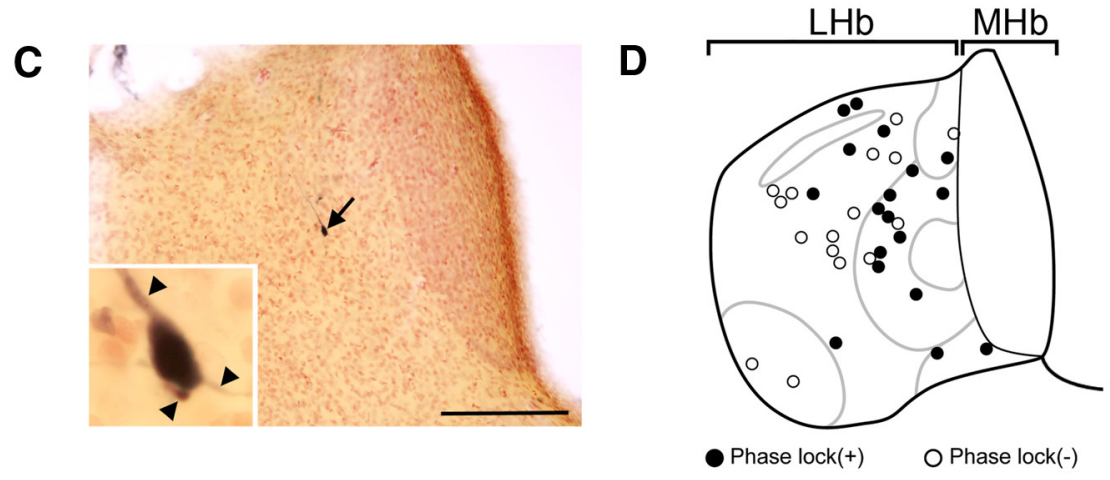

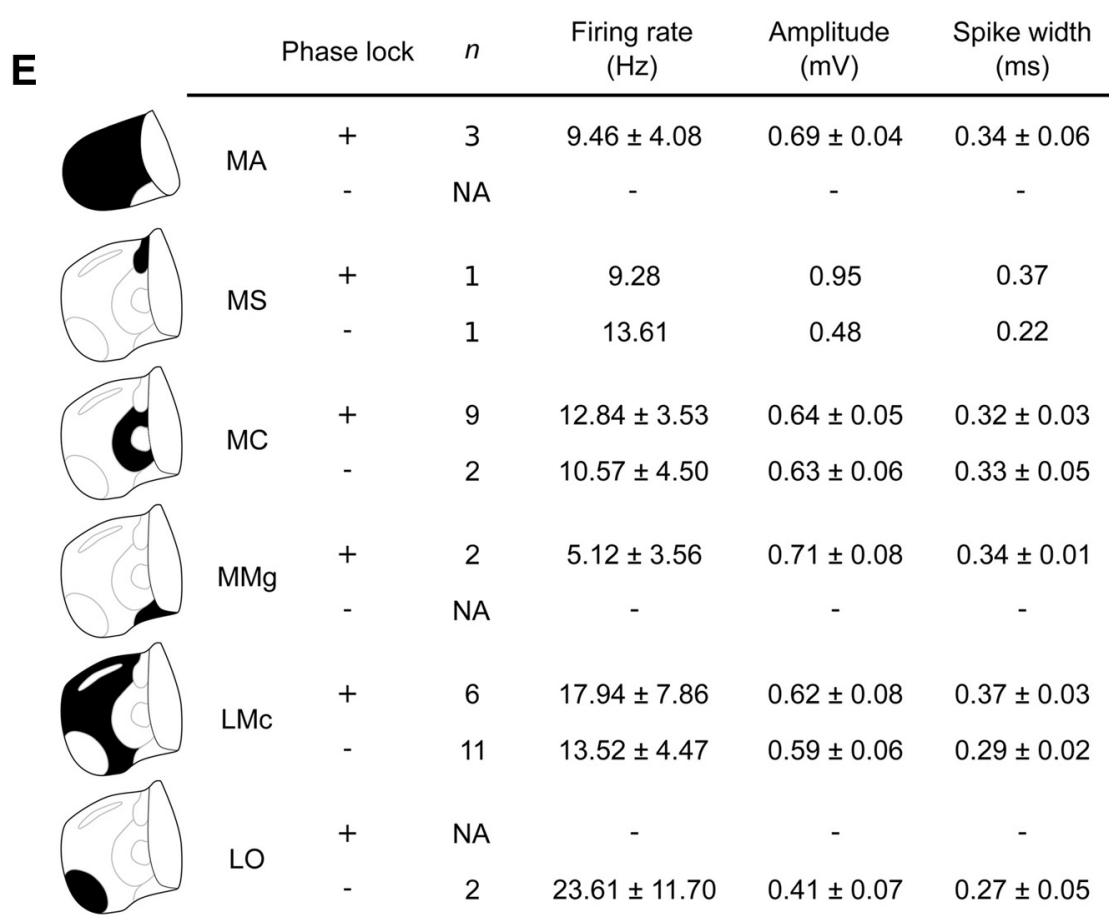

Figure 6. Specific localization of the phase-locking neurons in the medial division of the lateral habenula revealed by juxtacellular labeling. $A$, A representative activity of the lateral habenular neurons (red at the bottom) showing the preferential firing around the positive peak of the hippocampal theta oscillation (blue at the top, raw trace; blue at the middle, filtered trace between 3 and $6 \mathrm{~Hz}$ ). $\boldsymbol{B}$, Average spike shape of the neurons shown in $\boldsymbol{A}$. C, A coronal section of the habenula showing the localization of a labeled neuron presented in $\boldsymbol{A}$ (black arrow). Inset shows the magnified view of cell body of a labeled neuron with three dendrites (arrowheads). $\boldsymbol{D}$, Schematic diagram of the coronal section of the rat habenula showing the distribution of the recorded neurons identified by juxtacellular labeling. Black and white filled circles indicate the neurons with and without phase-locking activity, respectively. E, Summary table of the phase-locking activity, number of recorded neurons, firing rate, amplitude, and half-width of the spikes of the recorded neurons in the six subnuclei of the lateral habenula. Values are presented as mean \pm SEM. LHbMA, anterior part of LHbM; LHbMMg, marginal part of LHbM; LHbMS, superior part of LHbM; LHbL 0 , oval part of LHbL; NA, not available. Scale bar, $200 \mu \mathrm{m}$.

ized more frequently in the LHbM ( 15 of 18 cells) than in the LHbL ( 6 of 19 cells) ( $p<0.01$, Fisher's exact test; Fig. $6 D, E)$.

This analysis revealed that the neurons with phase-locking activity were not uniformly distributed across the entire area of 
LHb, but were concentrated particularly in LHbM. Since LHbM, in comparison with LHbL, preferentially receives inputs from the DBB region, which has pace-making activity toward the hippocampal theta oscillation (Herkenham and Nauta, 1977), these observations suggested that the DBB can act as a common source of GABAergic input to LHb and HPC to pace the phase-locking activity of the neurons in those structures in accordance with the ongoing theta oscillation in HPC.

\section{Discussion}

This study presented the first evidence that LHb neurons show coordinated activity with the fluctuating theta oscillation in HPC, and that intact $\mathrm{LHb}$ is needed to maintain the hippocampal theta oscillation under anesthesia and during REM sleep. Below, we discuss how the neural activity in LHb might work in coordination with changes in the brain states and the functional significance of this pathway in regulating the hippocampal theta oscillation.

\section{Lateral habenula as a regulatory region of the hippocampal theta}

Interaction between the habenula and hippocampus has been reported despite the anatomical distance. For example, destruction of both $\mathrm{LHb}$ and $\mathrm{MHb}$ leads to impairment of the hippocampus-dependent spatial memory as observed in the Morris water maze task (Lecourtier et al., 2004), and electrical stimulation of LHb induces activation of the pyramidal cell in HPC via serotonergic modulation (Sabatino et al., 1987). Theta power recorded around the hilus of the dentate gyrus was also decreased by lesioning of the fasciculus retroflexus, which contains the efferent projections from $\mathrm{MHb}$ and $\mathrm{LHb}$ as well as axons originating from the basal forebrain and passing through the habenular region (Valjakka et al., 1998). Furthermore, lesions in the interpeduncular nucleus, which primarily receives efferents from $\mathrm{MHb}$, resulted in shortened REM sleep in rat (Haun et al., 1992). Nevertheless, it remains unknown how and when LHb interacts with the hippocampus.

The electrophysiological analyses in the present study addressed this issue by revealing a fine temporal association of the neuronal firing in LHb with the oscillatory activity in HPC. This finding indicated that LHb is not simply a negative regulator of monoaminergic neurons, but that it could actually determine the timing by which this monoaminergic activity should be upregulated in coordination with specific brain states such as REM sleep. Using a specific lesion of LHb, we provided direct evidence for the involvement of $\mathrm{LHb}$ in regulating hippocampal theta oscillation.

\section{Mechanism responsible for phase locking in the lateral habenula}

Previous studies identified several brain regions with pacemaking activity that could play roles in regulating the theta oscillation in HPC (Vertes and Kocsis, 1997). One major source is the MS and vDBB. Although previous literature reported that LHbM receives preferential inputs from $\mathrm{vDBB}$ (Herkenham and Nauta, 1977), it remains unclear whether this pathway contributes to regulating the hippocampal theta, since previous experiments did not specify whether the horizontal or vertical limb of DBB sends the afferents to the LHbM. Our present analysis clarified the involvement of this pathway in the theta regulation by showing the intermingled distribution of GABAergic and cholinergic neurons in the $\mathrm{VDBB}$. Based on suggestions that the theta activity is formed by the buildup of a mutual inhibitory loop between the septal pacemaker and the HPC (Hangya et al., 2009), our results showing that the hippocampal theta leads the phase-locking firing in LHb support the idea that synchronous activity in the septo-hippocampal system entrains the LHb. It is unlikely that LHb directly drives the theta activity in the septo-hippocampal system due to the absence of projection from LHb to HPC or the pace-making septal region.

During theta oscillation in the hippocampus, pacemaker neurons in MS and $\mathrm{vDBB}$ were subdivided into several groups according to the preferred phase of the hippocampal theta cycle (Dragoi et al., 1999; Borhegyi et al., 2004). Since we identified two groups of LHb neurons that each preferentially fired at distinct phases of the theta cycle, examining whether activity of $\mathrm{vDBB}$ neurons correlated with that of LHb neurons under anesthesia and during REM sleep will clarify the mechanism by which LHb neurons show phase-locking activity to the hippocampal theta oscillation.

\section{Neural substrates for regulation of the hippocampal theta oscillation via the lateral habenula}

Serotonergic neurons in the raphe nuclei fire with phase locking to the hippocampal theta (Viana Di Prisco et al., 2002; Kocsis et al., 2006), and stimulation of the raphe consistently reduces the hippocampal theta (Vertes, 1981). Indeed, a recent study reported that changes in serotonergic neurotransmission underlie the fast control of neuronal activity in the hippocampal circuits through serotonergic projections from the median raphe terminating directly onto the hippocampal interneurons (Varga et al., 2009).

Previous studies also suggested that LHb affects the release of serotonin in the brain, although the direction of changes in firing rate of the serotonergic neurons might vary dependent upon the frequency and length of LHb stimulation (Wang and Aghajanian, 1977; Ferraro et al., 1996). Since LHbM output specifically targets the median raphe (Herkenham and Nauta, 1979; Bernard and Veh, 2012), and LHb stimulation affects the release of serotonin in the brain (Nishikawa and Scatton, 1985; Kalén et al., 1989), phase locking of serotonergic activity to the hippocampal theta oscillation (Kocsis et al., 2006) might be regulated by the LHb oscillatory activity observed in the present study. Therefore, it is interesting to consider that the emergence of such synchronous firing in the habenula-raphe pathway globally modulates the neural activities in the brain regions that show theta oscillation during animal behaviors contingent with emotional experience such as fear (Seidenbecher et al., 2003; Adhikari et al., 2010; Narayanan et al., 2011).

LHb neurons might also regulate the hippocampal theta during REM sleep with tonic firing as observed in NREM sleep (Fig. $2 C)$. However, our results excluded this possibility with the absence of firing rate changes across transitions between NREM and REM sleep (Fig. 3G). Indeed, the emergence of phase-locking activity in LHb specifically during REM sleep instead supports the hypothesis that LHb regulates hippocampal theta via its oscillatory activity.

Thus, our results revealed that the inhibitory effect of an LHb lesion on maintenance of the hippocampal theta was mediated via the intact serotonergic median raphe, suggesting that serotonergic raphe nuclei are primary candidates as the output target of LHb in regulating the hippocampal theta rhythm.

Another molecule that can mediate the modulatory effect of LHb on hippocampal theta oscillation is the neuropeptide relaxin-3, which is produced by neurons in the nucleus incertus. $\mathrm{LHb}$ projects directly to the nucleus incertus, which, in turn, sends axons to a variety of brain regions such as MS (Goto et al., 
2001; Olucha-Bordonau et al., 2003). Recent studies revealed that administering relaxin-3 protein to MS induces hippocampal theta oscillation in the awake condition, implicating a role for relaxin-3 as a modulator of the theta oscillation in HPC (Ma et al., 2009). Examining whether the serotonergic neurons in the raphe nuclei or the neurons expressing relaxin-3 in the nucleus incertus are under the influence of phase-locking activity in LHb would unravel the mechanism by which LHb regulates the hippocampal activity with fine temporal precision as observed in the present study.

It is also possible that neural activity in LHbM precedes and regulates the theta via structures further upstream of MS-DBB such as the supramammillary nucleus, which receives projections from LHbM (Kiss et al., 2002). It is less likely, however, that this pathway plays a major role in the habenular regulation of the theta, since our $Z$-shift analysis revealed that most of the phaselocking activity in LHbM neurons maximally fitted the past of the theta, i.e., LHbM activity followed the hippocampal theta.

\section{Functional implications of the coordinated activity of the lateral habenula and the hippocampus through the theta rhythm}

Theta oscillation in the hippocampus is associated not only with REM sleep, but also with other behaviors such as locomotion, in which theta oscillation in the hippocampus plays a critical role in encoding spatial information (Buzsáki, 2002). It is likely that LHb neurons also show phase-locking activity to the theta wave generated in exploration, since a subpopulation of LHb can fire at the range of theta frequency in the rats with locomotor activity (Sharp et al., 2006). More recently, theta activities in the ventral hippocampus and other regions such as prefrontal cortex or lateral amygdala showed transient synchronization with high coherence, when the animals were in the condition associated with emotional experiences such as anxiety (Adhikari et al., 2010) and fear (Seidenbecher et al., 2003). Considering that serotonergic raphe nuclei, which receive inhibitory input from the LHb, project to diverse brain regions including prefrontal cortex, lateral amygdala, and hippocampus (Vertes, 1991; Vertes et al., 1999), changes in firing pattern in $\mathrm{LHb}$ neurons might induce the synchronization of neural activities in those distant regions to facilitate neural processing between these brain regions when the animals are anxious or fearful.

Finally, dopaminergic neurons in VTA during REM sleep tend to fire more frequently in burst mode than in tonic mode (Dahan et al., 2007). Our results showed that the phase-locking activity in LHbM was observed specifically during REM sleep and lasted during those periods associated with hippocampal theta, suggesting that the lateral habenular pathway could also mediate the temporal regulation of dopaminergic neurons as well as the serotonergic neurons under regulation from the septo-hippocampal system. Our study supports the idea that firing pattern changes in LHbM induce a transition of this firing mode of midbrain dopaminergic neurons, and that this could serve as a synaptic mechanism for memory consolidation during REM sleep (Diekelmann and Born, 2010).

\section{References}

Adhikari A, Topiwala MA, Gordon JA (2010) Synchronized activity between the ventral hippocampus and the medial prefrontal cortex during anxiety. Neuron 65:257-269. CrossRef Medline

Aizawa H, Amo R, Okamoto H (2011) Phylogeny and ontogeny of the habenular structure. Front Neurosci 5:138. CrossRef Medline

Aizawa H, Kobayashi M, Tanaka S, Fukai T, Okamoto H (2012) Molecular characterization of the subnuclei in rat habenula. J Comp Neurol 520: 4051-4066. CrossRef Medline

Ambrosini MV, Mariucci G, Colarieti L, Bruschelli G, Carobi C, Giuditta A (1993) The structure of sleep is related to the learning ability of rats. Eur J Neurosci 5:269-275. CrossRef Medline

Amo R, Aizawa H, Takahoko M, Kobayashi M, Takahashi R, Aoki T, Okamoto H (2010) Identification of the zebrafish ventral habenula as a homolog of the mammalian lateral habenula. J Neurosci 30:1566-1574. CrossRef Medline

Andres KH, von Düring M, Veh RW (1999) Subnuclear organization of the rat habenular complexes. J Comp Neurol 407:130-150. CrossRef Medline

Berens P (2009) CircStat: a MATLAB toolbox for circular statistics. J Stat Softw 31:1-21.

Bernard R, Veh RW (2012) Individual neurons in the rat lateral habenular complex project mostly to the dopaminergic ventral tegmental area or to the serotonergic raphe nuclei. J Comp Neurol 520:2545-2558. CrossRef Medline

Borhegyi Z, Varga V, Szilágyi N, Fabo D, Freund TF (2004) Phase segregation of medial septal GABAergic neurons during hippocampal theta activity. J Neurosci 24:8470-8479. CrossRef Medline

Buzsáki G (2002) Theta oscillations in the hippocampus. Neuron 33:325340. CrossRef Medline

Christoph GR, Leonzio RJ, Wilcox KS (1986) Stimulation of the lateral habenula inhibits dopamine-containing neurons in the substantia nigra and ventral tegmental area of the rat. J Neurosci 6:613-619. Medline

Costa-Miserachs D, Portell-Cortés I, Torras-Garcia M, Morgado-Bernal I (2003) Automated sleep staging in rat with a standard spreadsheet. J Neurosci Methods 130:93-101. CrossRef Medline

Csicsvari J, Hirase H, Czurkó A, Mamiya A, Buzsáki G (1999) Oscillatory coupling of hippocampal pyramidal cells and interneurons in the behaving rat. J Neurosci 19:274-287. Medline

Dahan L, Astier B, Vautrelle N, Urbain N, Kocsis B, Chouvet G (2007) Prominent burst firing of dopaminergic neurons in the ventral tegmental area during paradoxical sleep. Neuropsychopharmacology 32:1232-1241. CrossRef Medline

Diekelmann S, Born J (2010) The memory function of sleep. Nat Rev Neurosci 11:114-126. CrossRef Medline

Dragoi G, Carpi D, Recce M, Csicsvari J, Buzsáki G (1999) Interactions between hippocampus and medial septum during sharp waves and theta oscillation in the behaving rat. J Neurosci 19:6191-6199. Medline

Ellison G (1994) Stimulant-induced psychosis, the dopamine theory of schizophrenia, and the habenula. Brain Res Brain Res Rev 19:223-239. CrossRef Medline

Ferraro G, Montalbano ME, Sardo P, La Grutta V (1996) Lateral habenular influence on dorsal raphe neurons. Brain Res Bull 41:47-52. CrossRef Medline

Fisher N (1993) Statistical analysis of circular data. Cambridge, UK: Cambridge UP.

Geisler S, Andres KH, Veh RW (2003) Morphologic and cytochemical criteria for the identification and delineation of individual subnuclei within the lateral habenular complex of the rat. J Comp Neurol 458:78-97. CrossRef Medline

Goto M, Swanson LW, Canteras NS (2001) Connections of the nucleus incertus. J Comp Neurol 438:86-122. CrossRef Medline

Green J, Arduini A (1954) Hippocampal electrical activity in arousal. J Neurophysiol 17:533-557. Medline

Hangya B, Borhegyi Z, Szilágyi N, Freund TF, Varga V (2009) GABAergic neurons of the medial septum lead the hippocampal network during theta activity. J Neurosci 29:8094-8102. CrossRef Medline

Haun F, Eckenrode TC, Murray M (1992) Habenula and thalamus cell transplants restore normal sleep behaviors disrupted by denervation of the interpeduncular nucleus. J Neurosci 12:3282-3290. Medline

Hazan L, Zugaro M, Buzsáki G (2006) Klusters, NeuroScope, NDManager: a free software suite for neurophysiological data processing and visualization. J Neurosci Methods 155:207-216. CrossRef Medline

Herkenham M, Nauta WJ (1977) Afferent connections of the habenular nuclei in the rat. A horseradish peroxidase study, with a note on the fiber-of-passage problem. J Comp Neurol 173:123-146. CrossRef Medline

Herkenham M, Nauta WJ (1979) Efferent connections of the habenular nuclei in the rat. J Comp Neurol 187:19-47. CrossRef Medline

Hobson JA, McCarley RW, Wyzinski PW (1975) Sleep cycle oscillation: re- 
ciprocal discharge by two brainstem neuronal groups. Science 189:55-58. CrossRef Medline

Isomura Y, Harukuni R, Takekawa T, Aizawa H, Fukai T (2009) Microcircuitry coordination of cortical motor information in self-initiation of voluntary movements. Nat Neurosci 12:1586-1593. CrossRef Medline

Jouvet M (1969) Biogenic amines and the states of sleep. Science 163:32-41. CrossRef Medline

Kalén P, Strecker RE, Rosengren E, Björklund A (1989) Regulation of striatal serotonin release by the lateral habenula-dorsal raphe pathway in the rat as demonstrated by in vivo microdialysis: role of excitatory amino acids and GABA. Brain Res 492:187-202. CrossRef Medline

Kim U, Chang SY (2005) Dendritic morphology, local circuitry, and intrinsic electrophysiology of neurons in the rat medial and lateral habenular nuclei of the epithalamus. J Comp Neurol 483:236-250. CrossRef Medline

Kiss J, Csáki A, Bokor H, Kocsis K, Kocsis B (2002) Possible glutamatergic/ aspartatergic projections to the supramammillary nucleus and their origins in the rat studied by selective [(3)H]D-aspartate labelling and immunocytochemistry. Neuroscience 111:671-691. CrossRef Medline

Klausberger T, Somogyi P (2008) Neuronal diversity and temporal dynamics: the unity of hippocampal circuit operations. Science 321:53-57. CrossRef Medline

Klausberger T, Magill PJ, Márton LF, Roberts JD, Cobden PM, Buzsáki G, Somogyi P (2003) Brain-state- and cell-type-specific firing of hippocampal interneurons in vivo. Nature 421:844-848. CrossRef Medline

Klemm WR (2004) Habenular and interpeduncularis nuclei: shared components in multiple-function networks. Med Sci Monit 10:RA261-RA273. Medline

Kocsis B, Varga V, Dahan L, Sik A (2006) Serotonergic neuron diversity: identification of raphe neurons with discharges time-locked to the hippocampal theta rhythm. Proc Natl Acad Sci U S A 103:1059-1064. CrossRef Medline

Kowski AB, Veh RW, Weiss T (2009) Dopaminergic activation excites rat lateral habenular neurons in vivo. Neuroscience 161:1154-1165. CrossRef Medline

Kramis R, Vanderwolf CH, Bland BH (1975) Two types of hippocampal rhythmical slow activity in both the rabbit and the rat: relations to behavior and effects of atropine, diethyl ether, urethane, and pentobarbital. Exp Neurol 49:58-85. CrossRef Medline

Lecourtier L, Kelly PH (2005) Bilateral lesions of the habenula induce attentional disturbances in rats. Neuropsychopharmacology 30:484-496. CrossRef Medline

Lecourtier L, Neijt HC, Kelly PH (2004) Habenula lesions cause impaired cognitive performance in rats: implications for schizophrenia. Eur J Neurosci 19:2551-2560. CrossRef Medline

Li B, Piriz J, Mirrione M, Chung C, Proulx CD, Schulz D, Henn F, Malinow R (2011) Synaptic potentiation onto habenula neurons in the learned helplessness model of depression. Nature 470:535-539. CrossRef Medline

Limebeer CL, Parker LA, Fletcher PJ (2004) 5,7-dihydroxytryptamine lesions of the dorsal and median raphe nuclei interfere with lithiuminduced conditioned gaping, but not conditioned taste avoidance, in rats. Behav Neurosci 118:1391-1399. CrossRef Medline

Ma S, Olucha-Bordonau FE, Hossain MA, Lin F, Kuei C, Liu C, Wade JD, Sutton SW, Nuñez A, Gundlach AL (2009) Modulation of hippocampal theta oscillations and spatial memory by relaxin-3 neurons of the nucleus incertus. Learn Mem 16:730-742. CrossRef Medline

Matsumoto M, Hikosaka O (2007) Lateral habenula as a source of negative reward signals in dopamine neurons. Nature 447:1111-1115. CrossRef Medline

McHugh TJ, Blum KI, Tsien JZ, Tonegawa S, Wilson MA (1996) Impaired hippocampal representation of space in CA1-specific NMDAR1 knockout mice. Cell 87:1339-1349. CrossRef Medline

Narayanan V, Heiming RS, Jansen F, Lesting J, Sachser N, Pape HC, Seidenbecher T (2011) Social defeat: impact on fear extinction and amygdalaprefrontal cortical theta synchrony in 5-HTT deficient mice. PLoS One 6:e22600. CrossRef Medline

Nishikawa T, Scatton B (1985) Inhibitory influence of GABA on central serotonergic transmission. Involvement of the habenulo-raphe pathways in the GABAergic inhibition of ascending cerebral serotonergic neurons. Brain Res 331:81-90. CrossRef Medline

Nishikawa T, Fage D, Scatton B (1986) Evidence for, and nature of, the tonic inhibitory influence of habenulointerpeduncular pathways upon cerebral dopaminergic transmission in the rat. Brain Res 373:324-336. CrossRef Medline

Olucha-Bordonau FE, Teruel V, Barcia-González J, Ruiz-Torner A, ValverdeNavarro AA, Martínez-Soriano F (2003) Cytoarchitecture and efferent projections of the nucleus incertus of the rat. J Comp Neurol 464:62-97. CrossRef Medline

Petsche H, Stumpf C, Gogolak G (1962) The significance of the rabbit's septum as a relay station between the midbrain and the hippocampus. I. The control of hippocampus arousal activity by the septum cells. Electroencephalogr Clin Neurophysiol 14:202-211. CrossRef Medline

Pinault D (1996) A novel single-cell staining procedure performed in vivo under electrophysiological control: morpho-functional features of juxtacellularly labeled thalamic cells and other central neurons with biocytin or Neurobiotin. J Neurosci Methods 65:113-136. CrossRef Medline

Rutishauser U, Ross IB, Mamelak AN, Schuman EM (2010) Human memory strength is predicted by theta-frequency phase-locking of single neurons. Nature 464:903-907. CrossRef Medline

Sabatino M, Ferraro G, Vella N, La Grutta V (1987) An electrophysiological study of habenular influence on hippocampus. Neurosci Lett 78:75-79. CrossRef Medline

Sartorius A, Kiening KL, Kirsch P, von Gall CC, Haberkorn U, Unterberg AW, Henn FA, Meyer-Lindenberg A (2010) Remission of major depression under deep brain stimulation of the lateral habenula in a therapyrefractory patient. Biol Psychiatry 67:e9-e11. CrossRef Medline

Seidenbecher T, Laxmi TR, Stork O, Pape HC (2003) Amygdalar and hippocampal theta rhythm synchronization during fear memory retrieval. Science 301:846-850. CrossRef Medline

Sharp PE, Turner-Williams S, Tuttle S (2006) Movement-related correlates of single cell activity in the interpeduncular nucleus and habenula of the rat during a pellet-chasing task. Behav Brain Res 166:55-70. CrossRef Medline

Siapas AG, Lubenov EV, Wilson MA (2005) Prefrontal phase locking to hippocampal theta oscillations. Neuron 46:141-151. CrossRef Medline

Takekawa T, Isomura Y, Fukai T (2010) Accurate spike sorting for multiunit recordings. Eur J Neurosci 31:263-272. CrossRef Medline

Valjakka A, Vartiainen J, Tuomisto L, Tuomisto JT, Olkkonen H, Airaksinen MM (1998) The fasciculus retroflexus controls the integrity of REM sleep by supporting the generation of hippocampal theta rhythm and rapid eye movements in rats. Brain Res Bull 47:171-184. CrossRef Medline

Vanderwolf CH, Leung LW, Baker GB, Stewart DJ (1989) The role of serotonin in the control of cerebral activity: studies with intracerebral 5,7dihydroxytryptamine. Brain Res 504:181-191. CrossRef Medline

Varga V, Losonczy A, Zemelman BV, Borhegyi Z, Nyiri G, Domonkos A, Hangya B, Holderith N, Magee JC, Freund TF (2009) Fast synaptic subcortical control of hippocampal circuits. Science 326:449-453. CrossRef Medline

Vertes RP (1981) An analysis of ascending brain stem systems involved in hippocampal synchronization and desynchronization. J Neurophysiol 46: 1140-1159. Medline

Vertes RP (1991) A PHA-L analysis of ascending projections of the dorsal raphe nucleus in the rat. J Comp Neurol 313:643-668. CrossRef Medline

Vertes RP (2005) Hippocampal theta rhythm: a tag for short-term memory. Hippocampus 15:923-935. CrossRef Medline

Vertes RP, Kocsis B (1997) Brainstem-diencephalo-septohippocampal systems controlling the theta rhythm of the hippocampus. Neuroscience 81:893-926. CrossRef Medline

Vertes RP, Kinney GG, Kocsis B, Fortin WJ (1994) Pharmacological suppression of the median raphe nucleus with serotonin1A agonists, 8-OHDPAT and buspirone, produces hippocampal theta rhythm in the rat. Neuroscience 60:441-451. CrossRef Medline

Vertes RP, Fortin WJ, Crane AM (1999) Projections of the median raphe nucleus in the rat. J Comp Neurol 407:555-582. CrossRef Medline

Viana Di Prisco G, Albo Z, Vertes RP, Kocsis B (2002) Discharge properties of neurons of the median raphe nucleus during hippocampal theta rhythm in the rat. Exp Brain Res 145:383-394. CrossRef Medline

Wang RY, Aghajanian GK (1977) Physiological evidence for habenula as major link between forebrain and midbrain raphe. Science 197:89-91. CrossRef Medline

Wilcox KS, Gutnick MJ, Christoph GR (1988) Electrophysiological properties of neurons in the lateral habenula nucleus: an in vitro study. J Neurophysiol 59:212-225. Medline 
Wyss JM, Swanson LW, Cowan WM (1979) A study of subcortical afferents to the hippocampal formation in the rat. Neuroscience 4:463-476. CrossRef Medline
Zhao H, Rusak B (2005) Circadian firing-rate rhythms and light responses of rat habenular nucleus neurons in vivo and in vitro. Neuroscience 132: 519-528. CrossRef Medline 